ARTICLE

\title{
LPA signaling acts as a cell-extrinsic mechanism to initiate cilia disassembly and promote neurogenesis
}

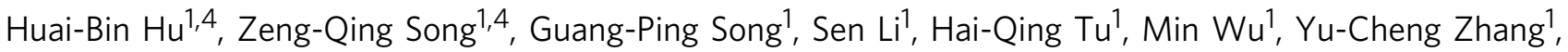
Jin-Feng Yuan ${ }^{1}$, Ting-Ting Li ${ }^{1}$, Pei-Yao Li ${ }^{1}$, Yu-Ling Xu' ${ }^{1}$ Xiao-Lin Shen ${ }^{1}$, Qiu-Ying Han ${ }^{1}$, Ai-Ling Li ${ }^{1}$, Tao Zhou', Jerold Chun (1) ${ }^{2}$, Xue-Min Zhang (i) ${ }^{1 \times}$ \& Hui-Yan Li ${ }^{1,3 凶}$

Dynamic assembly and disassembly of primary cilia controls embryonic development and tissue homeostasis. Dysregulation of ciliogenesis causes human developmental diseases termed ciliopathies. Cell-intrinsic regulatory mechanisms of cilia disassembly have been wellstudied. The extracellular cues controlling cilia disassembly remain elusive, however. Here, we show that lysophosphatidic acid (LPA), a multifunctional bioactive phospholipid, acts as a physiological extracellular factor to initiate cilia disassembly and promote neurogenesis. Through systematic analysis of serum components, we identify a small molecular-LPA as the major driver of cilia disassembly. Genetic inactivation and pharmacological inhibition of LPA receptor 1 (LPAR1) abrogate cilia disassembly triggered by serum. The LPA-LPAR-Gprotein pathway promotes the transcription and phosphorylation of cilia disassembly factorsAurora A, through activating the transcription coactivators YAP/TAZ and calcium/CaM pathway, respectively. Deletion of Lpar1 in mice causes abnormally elongated cilia and decreased proliferation in neural progenitor cells, thereby resulting in defective neurogenesis. Collectively, our findings establish LPA as a physiological initiator of cilia disassembly and suggest targeting the metabolism of LPA and the LPA pathway as potential therapies for diseases with dysfunctional ciliogenesis.

\footnotetext{
${ }^{1}$ State Key Laboratory of Proteomics, National Center of Biomedical Analysis, Beijing, China. ${ }^{2}$ Sanford Burnham Prebys Medical Discovery Institute, La Jolla, USA. ${ }^{3}$ School of Basic Medical Sciences, Fudan University, Shanghai, China. ${ }^{4}$ These authors contributed equally: Huai-Bin Hu, Zeng-Qing Song.

凶email: zhangxuemin@cashq.ac.cn; hyli@ncba.ac.cn
} 
T he primary cilia are antenna-like organelles assembled on the surface of most mammalian cells $\mathbf{s}^{1,2}$. They act as physical and chemical sensors and transducers of extracellular signals, and play critical roles in diverse cellular processes, including embryonic development and tissue homeostasis ${ }^{3-6}$. Cilia dysfunction is linked to tumorigenesis and numerous human developmental diseases collectively called ciliopathies ${ }^{7-11}$.

Ciliogenesis is dynamically controlled by cilia assembly and disassembly. Cilia assembly occurs in non-dividing (G0/G1) cells in response to serum deprivation, whereas cilia must be disassembled prior to cell cycle re-entry upon serum stimulation ${ }^{12,13}$. Many regulators of the cell cycle, such as Aurora kinase A (Aurora A), NIMA-related kinases (NEK) and Polo-like kinase 1 (PLK1), have emerged as crucial modulators of cilia disassembly ${ }^{13-15}$. Therefore, cilia disassembly is tightly coupled with cell cycle.

PDGF and IGF-1 at concentrations much higher than those in serum can induce cilia disassembly to a degree, but they do so much less efficiently than serum itself ${ }^{13,16,17}$. Thus, the major component in serum that induces cilia disassembly is unknown. Dynamic ciliogenesis is critical for tissue differentiation, including neurogenesis ${ }^{18,19}$. However, the extracellular cues controlling cilia disassembly in vivo remain elusive.

During cortical development in mammals, neurogenesis occurs within two germinal zones of the embryonic cerebral cortex-the ventricular zone (VZ) and the subventricular zone $(\mathrm{SVZ})^{20}$. The neural progenitor cells (NPCs) from these two germinal zones generate projection neurons dedicated to all cortical layers ${ }^{21,22}$. The primary cilia in the radial glia (RG) cells in the VZ extend into the lateral ventricle and function as cellular antenna to detect signals present in the cerebrospinal fluid (CSF), thus regulating neurogenesis ${ }^{23-25}$. These cilia are highly dynamic. They are present during interphase and disassembled prior to entry into mitosis ${ }^{26-28}$. The mitotic entry of RG cells needs to be spatially and temporally regulated to coordinate their self-renewal divisions and asymmetric divisions to form the SVZ ${ }^{20,29}$. However, the developmental cues regulating cilia disassembly and mitotic entry of RG cells are unknown.

Lysophosphatidic acid (LPA), a multifunctional bioactive phospholipid, has been implicated in a variety of biological processes, including embryonic development and lymphocyte trafficking ${ }^{30-35}$. LPA is abundantly present in many tissues and in circulating blood ${ }^{36}$. It is mainly produced from lysophosphatidylcholine by the enzyme Autotaxin (Atx; Enpp2), a secreted lysophospholipase D (lysoPLD). LPA binds and activates LPA receptors (LPARs), an important class of G-proteincoupled receptors ${ }^{37}$. Upregulation of LPA signaling causes tumorigenesis ${ }^{38}$. Lpar1-depleted mice exhibited $\sim 50 \%$ neonatal lethality and adult Lpar1 ${ }^{-l-}$ survivors showing reduced size and craniofacial dysmorphism ${ }^{32}$.

In this study, we identify LPA as the major factor in serum for driving cilia disassembly. LPAR1 is critical for proper ciliogenesis, VZ/SVZ generation, and neocortex formation in mice. Our findings establish LPA as an important developmental cue of cilia disassembly, link defective ciliogenesis to diseases with elevated LPA signaling, and suggest chemical modulators of the LPA metabolism and LPA pathway as potential therapeutic agents for diseases with dysfunctional ciliogenesis.

\section{Results}

LPA is the major serum factor that triggers cilia disassembly. To identify the component in serum that can induce cilia disassembly, we set up a cilia disassembly assay with cultured human retinal pigment epithelial (RPE-1) cells ${ }^{13}$. Briefly, we serum-starved RPE-1 cells for $48 \mathrm{~h}$ to induce cilia formation in the majority of cells. Ciliated cells were then re-stimulated with serum for $24 \mathrm{~h}$ to trigger the disassembly of cilia (Fig. 1a). The effect of serum on cilia disassembly was dose-dependent (Fig. 1b). The minimum concentration of serum that could efficiently induce cilia disassembly was about 1\% (Fig. 1b and Supplementary Fig. 1a). Since growth factors such as PDGF (platelet-derived growth factor) and IGF-1 (insulin-like growth factor 1) have been reported to weakly induce ciliary disassembly $13,16,17$, we tested several major growth factors in serum, including PDGF, IGF, EGF and FGF, in cilia disassembly. As previously reported, only PDGF at high concentrations caused limited cilia disassembly (Supplementary Fig. 1b). Therefore, these growth factors are unlikely to be the major components in serum that trigger cilia disassembly.

To determine which component in serum is responsible for cilia disassembly, we next destroyed the protein and nucleic acid components in serum by boiling or treating with pronase or turbonuclease and then tested the cilia-disassembly activity of the treated serum. Interestingly, the cilia-disassembly activity was resistant to all these harsh treatments (Fig. 1c and Supplementary Fig. 1c). These results suggest that the cilia disassembly activity may not be caused by proteins or nucleic acids. We next removed lipids from serum and treated ciliated cells with this lipiddepleted serum. We found that lipid-depleted serum had a markedly decreased ability to induce cilia disassembly (Fig. 1c and Supplementary Fig. 1c). This observation suggests that the factor(s) triggering cilia disassembly in serum might be lipid molecules.

To identify which lipids in serum-induced cilia disassembly, we tested a series of phospholipids in our cilia disassembly assay. Among the phospholipids tested, only lysophosphatidic acid (LPA) strongly induced cilia disassembly in RPE-1 cells (Fig. 1d and Supplementary Fig. 1d). Various LPA isoforms with different lengths and degrees of saturation of the fatty acid tail could efficiently trigger cilia disassembly (Supplementary Fig. 1e). As 18:1-LPA is the most widely used isoform in the laboratory ${ }^{37}$, we used 18:1-LPA in all subsequent LPA treatment experiments. LPA is an abundant phospholipid in serum and its concentration in human serum is in the $\mu \mathrm{M}$ range $\mathrm{e}^{39,40}$. At this concentration, LPA alone could induce substantial cilia disassembly as revealed by dose response analysis (Fig. 1e, f), suggesting that LPA in serum is adequate to induce cilia disassembly. Given that cilia disassembly occurred in two waves after serum stimulation (1-2 h and $18-24 \mathrm{~h}$, respectively ${ }^{13}$, we next examined the disassembly waves induced by LPA treatment. Similar to serum, LPA treatment obviously induced cilia disassembly at 2,18 , and $24 \mathrm{~h}$ (Supplementary Fig. 1f). In addition, the cilia disassembly activity of LPA was also observed in multiple other cell lines, including MEF and IMCD3 (Fig. 1g and Supplementary Fig. 1g). Thus, these results indicate that LPA is the major component in serum to trigger cilia disassembly.

The LPA receptors (LPARs) are required for LPA-induced cilia disassembly. LPA acts through a family of high-affinity Gprotein-coupled receptors, LPAR1- ${ }^{41}$, only LPAR1 was highly expressed in RPE-1 cells (Fig. 2a). Knockdown of LPAR1 blocked the fetal bovine serum (FBS)- or LPA-induced cilia disassembly in RPE-1 cells (Fig. 2b, c). Serum or LPA treatment failed to induce the LPAR1-depleted cells to re-enter the cell cycle (Supplementary Fig. 2a). Importantly, the effect of FBS or LPA on cilia disassembly in ciliated, LPAR1-depleted RPE-1 cells was rescued by the expression of an small interfering RNA (siRNA)-resistant LPAR1 transgene (Fig. 2d, e and Supplementary Fig. 2b). Treatment of ciliated RPE-1 cells with Ki16425, an LPAR1 and LPAR3 antagonist ${ }^{42}$, markedly reduced serum- or LPA-induced cilia disassembly (Fig. 2f). These data indicate that the LPA-LPAR signal pathway is essential for serum- or LPA-triggered cilia 
a

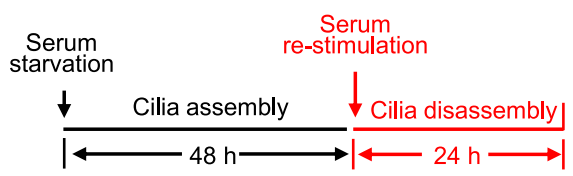

C

e
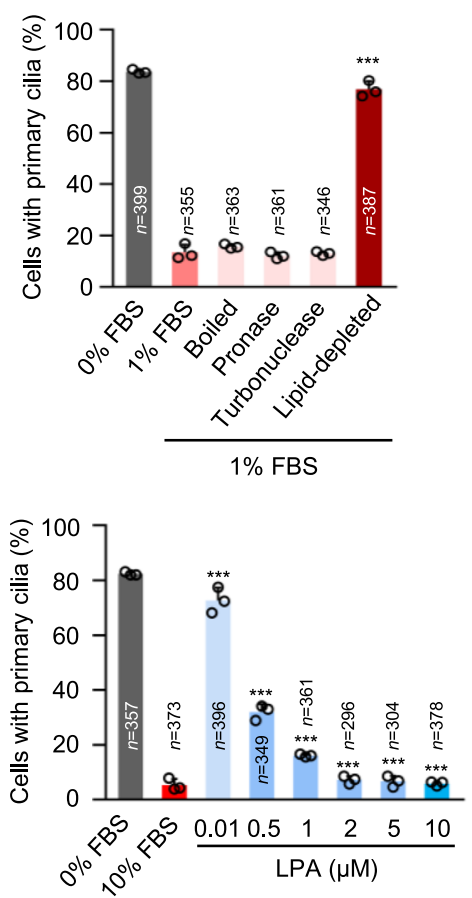

g b

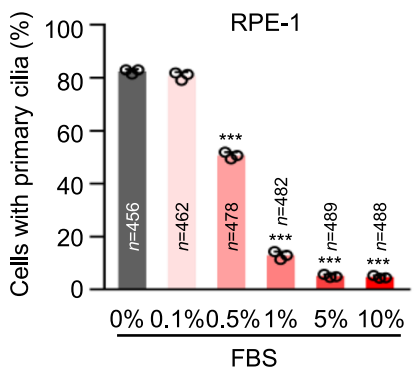

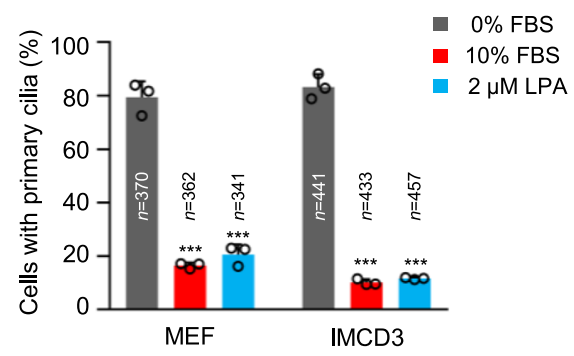

f
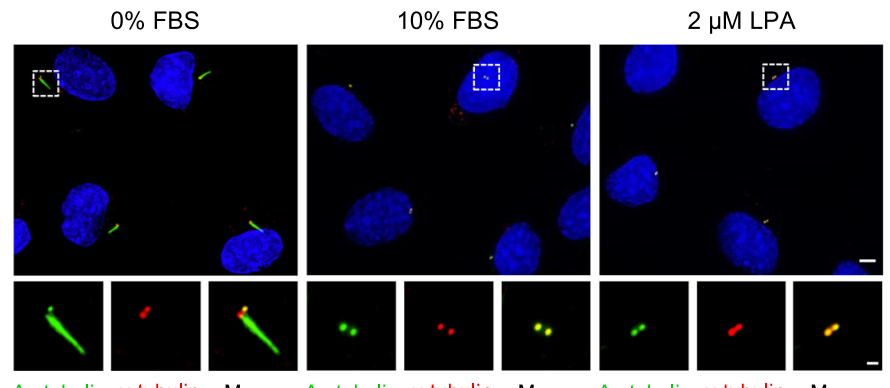

Fig. 1 Identification of LPA as the major cilia disassembly factor in serum. a Diagram indicates the timing of serum starvation (cilia assembly) and re-stimulation (cilia disassembly). b Serum induces cilia disassembly in a dose-dependent manner. Human retinal pigment epithelial (RPE-1) cells were starved in DMEM/F12 for $48 \mathrm{~h}$ to induce cilia formation, and then ciliated RPE-1 cells were re-stimulated with indicated concentrations of FBS for $24 \mathrm{~h}$ to induce cilia disassembly. c Lipids are responsible for serum-induced cilia disassembly. Ciliated RPE-1 cells (serum-starved) were stimulated with $1 \%$ boiled, pronase-pretreated, turbonuclease-pretreated or lipid-depleted serum for $24 \mathrm{~h}$, respectively. d Lysophosphatidic acid (LPA) induces cilia disassembly. Ciliated RPE-1 cells (serum-starved) were stimulated with $100 \mu \mathrm{M}$ of various phospholipids for $24 \mathrm{~h}$. Full name of lipids used are shown in "Methods." e Dose-dependent effects of LPA on cilia disassembly. Ciliated RPE-1 cells (serum-starved) were treated with different concentrations of LPA for $24 \mathrm{~h}$. $\mathbf{f}$ Representative images of RPE-1 cells in (e). Cells were stained with anti-Ac-tubulin (green) and anti- $\gamma$-tubulin (red) antibodies. Scale bar, $5 \mu \mathrm{m}$ (main image) and $1 \mu \mathrm{m}$ (magnified region). Three experiments were repeated independently with similar results. $\mathbf{g}$ LPA treatment ( $2 \mu \mathrm{M}$ for $24 \mathrm{~h}$ ) induces cilia disassembly in primary MEF and IMCD3 cells. Source data are provided as a Source Data file. Data are presented as mean \pm S.D. of three independent experiments in ( $\mathbf{b}-\mathbf{e})$ and $(\mathbf{g}) . n$, number of cells. ${ }^{\star \star \star} P<0.001$. One-way ANOVA test was performed followed by Dunnett's multiple comparisons in $\mathbf{b}$-e; two-way ANOVA test was performed in followed by Dunnett's multiple comparisons in $\mathbf{g}$. 
a

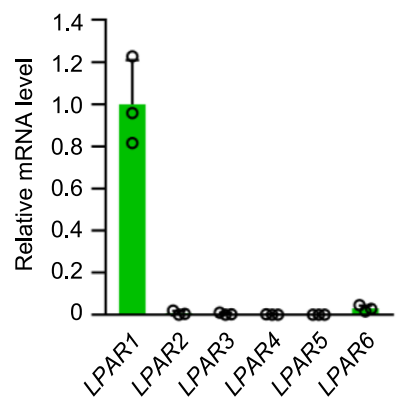

d
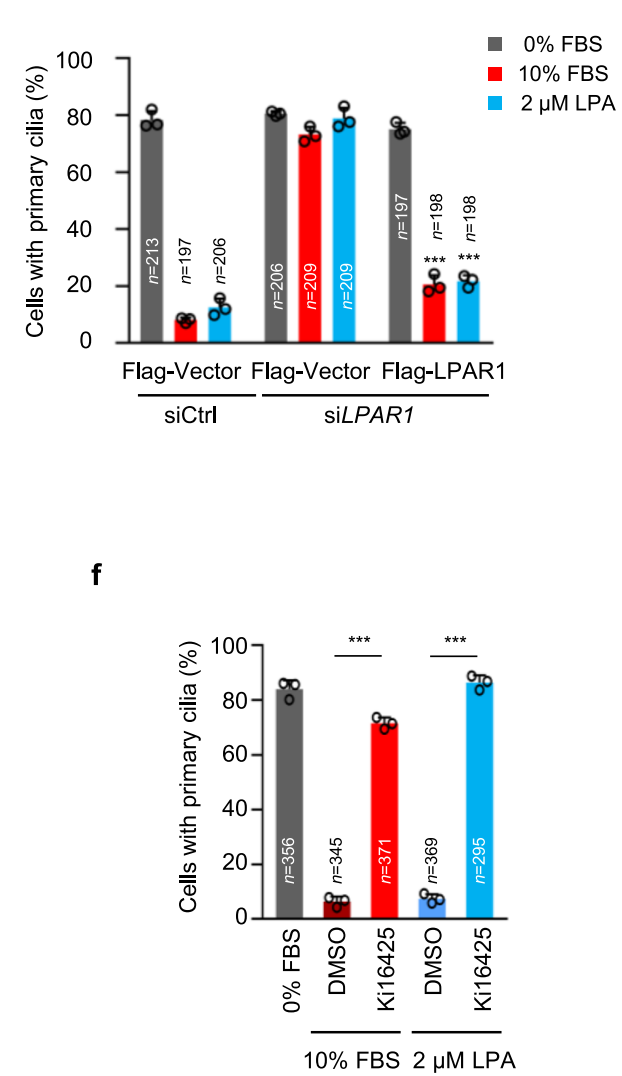

h
C

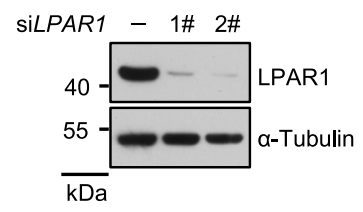

- $0 \% \mathrm{FBS}$

- $10 \% \mathrm{FBS}$

- $2 \mu \mathrm{M}$ LPA

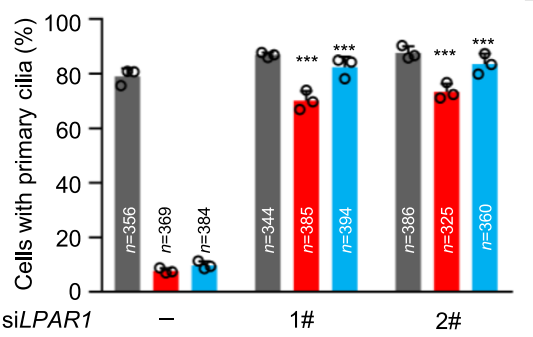

e

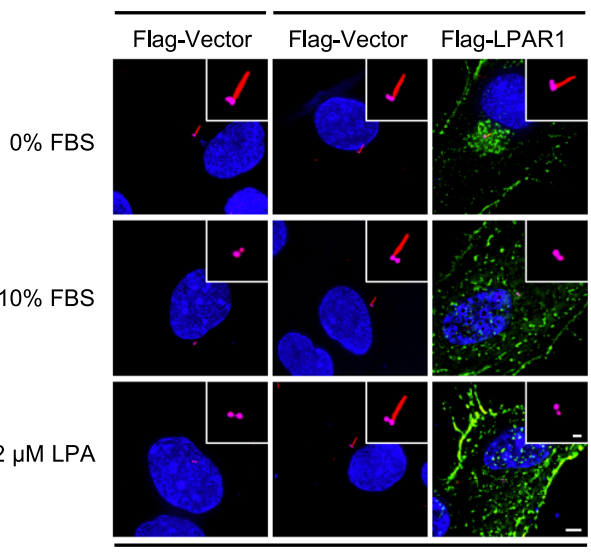

Flag + Ac-tubulin + y-tubulin

g

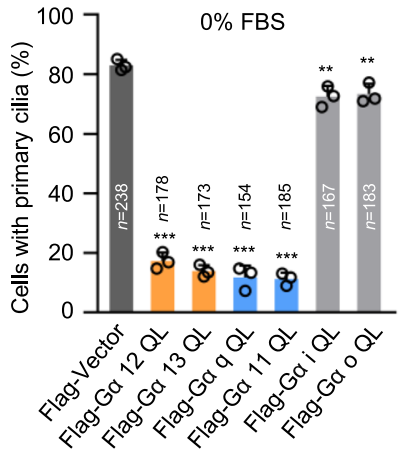

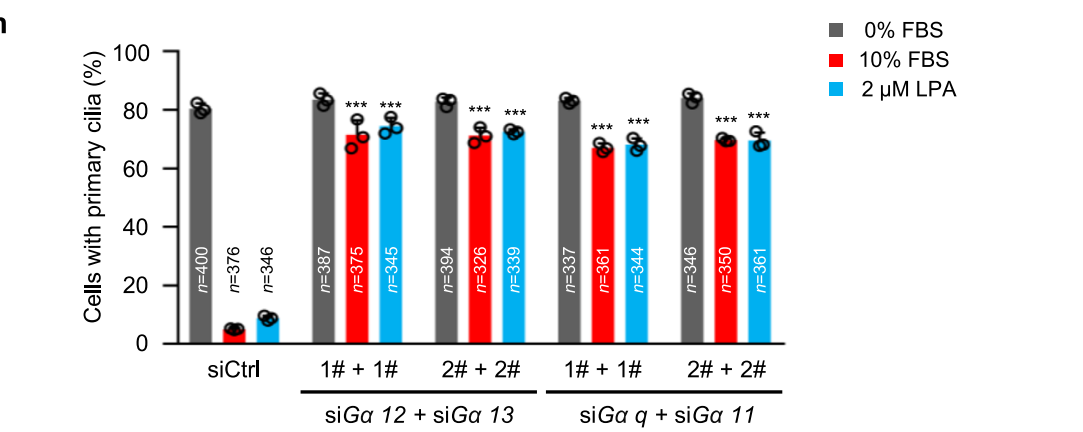

disassembly. Intriguingly, knockdown of LPAR1 or addition of Ki16425 in RPE cells directly resulted in cilia formation in cycling cells cultured with 10\% FBS (Supplementary Fig. 2c-e). One possibility is that the abolishment of the LPA inhibitory function might directly trigger the early events of cilium biogenesis, as suggested by a recent study ${ }^{43}$. Another possibility is that the assembly and disassembly of cilia are dynamically balanced processes. The disruption of disassembly can directly release the assembly activity. Consistent with this latter notion, the depletion of canonical disassembly factors, including Nek2 and Kif24, can also induce ciliogenesis in serum-cultured cells ${ }^{14}$.

LPARs bind to multiple heterotrimeric $G$ proteins, such as $\mathrm{Ga}$ 12/13, Ga q/11 and $\mathrm{Ga}$ i/o and activate various downstream signals that mediate cell migration and growth ${ }^{37,41}$. Overexpression of 
Fig. 2 The LPA-LPAR pathway promotes cilia disassembly. a Expression of LPA receptors (LPARs) in RPE-1 cells. The mRNA levels of LPAR1-6 were determined by quantitative PCR ( $9 P C R$ ). b, c Knockdown of LPAR1 blocks the effect of serum- and LPA-induced cilia disassembly. RPE-1 cells were starved for $12 \mathrm{~h}$ and then transfected with control siRNA or LPAR1 siRNAs respectively. Following $48 \mathrm{~h}$ serum starvation, cells were then treated with medium contained $10 \%$ FBS or $2 \mu \mathrm{M}$ LPA for $24 \mathrm{~h}$. b Immunoblotting shows the protein level of LPAR1 in LPAR1-knockdown RPE- 1 cells. $\alpha$-tubulin was used as a loading control. c Quantification of ciliation in RPE-1 cells. d, e Expression of Flag-LPAR1 resistant plasmid rescues cilia disassembly defects in LPAR1depleted cells. d Quantification of ciliation in RPE-1 cells. e Representative images of RPE-1 cells in d. Cells were stained with anti-Flag (green), anti- Actubulin (red) and anti- $\gamma$-tubulin (magenta) antibodies. Scale bar: $5 \mu \mathrm{m}$ (main image) and $1 \mu \mathrm{m}$ (magnified region). f LPAR1/3 antagonist Ki16425 blocks serum- and LPA-induced cilia disassembly. Ciliated RPE-1 cells were pretreated with Ki16425 (40 $\mu \mathrm{M})$ or DMSO control for 30 min, and then cells were stimulated with $10 \%$ FBS or $2 \mu \mathrm{M}$ LPA for $24 \mathrm{~h}$. $\mathbf{g}$ The effect of G $\alpha$ protein overexpression on cilia disassembly. RPE-1 cells were starved for $48 \mathrm{~h}$, and then transfected with Flag-G $\alpha$ plasmids expressing constitutively active $\mathrm{G} \alpha$ protein mutants (QL). $\mathbf{h}$ Knockdown of $\mathrm{G} \alpha 12 / 13$ or $\mathrm{G} \alpha \mathrm{q} / 11$ blocks the effect of serum- and LPA-induced cilia disassembly. RPE-1 cells were starved for $12 \mathrm{~h}$ and then transfected with control siRNA, a pool of siRNAs for G $\alpha 12$ and $\mathrm{G} \alpha 13$, or a pool of siRNAs for $G \alpha q$ and $G \alpha 11$, respectively. Following $48 \mathrm{~h}$ serum starvation, cells were treated with $10 \%$ FBS or $2 \mu \mathrm{M}$ LPA for $24 \mathrm{~h}$. Source data are provided as a Source Data file. Three experiments were repeated independently with similar results in $\mathbf{b}$ and $\mathbf{e}$. Data are presented as mean \pm S.D. of three independent experiments in $\mathbf{a}, \mathbf{c}, \mathbf{d}$ and $\mathbf{f}-\mathbf{h}$. $n$, number of cells. ${ }^{\star \star} P<0.01,{ }^{\star \star \star} p<0.001$. One-way ANOVA test was performed followed by Dunnett's multiple comparisons in $\mathbf{g}$ or followed by Bonferroni's multiple comparisons in $\mathbf{f}$; two-way ANOVA test was performed followed by Dunnett's multiple comparisons in $\mathbf{c}$, $\mathbf{d}$ and $\mathbf{h}$.

constitutively active mutants (QL) of Ga 12/13/q/11, but not Ga i/o, markedly induced cilia disassembly in RPE-1 cells, even when these cells were serum-starved (Fig. $2 \mathrm{~g}$ and Supplementary Fig. 2f, g). Conversely, the double knockdowns of Ga 12/13 or Ga q/11 blocked cilia disassembly in RPE-1 cells (Fig. $2 \mathrm{~h}$ and Supplementary Fig. 2h). These results suggest that the LPA-LPAR pathway promotes cilia disassembly mainly through Ga 12/13 and Ga q /11.

LPA pathway initiates cilia disassembly through Aurora A. To decipher the mechanism underlying how LPA-LPAR pathway promotes cilia disassembly, we performed RNA-Seq to analyze the transcriptome changes in control and LPAR1-depleted cells with or without LPA treatment. By comparing gene expression levels in LPA-untreated or -treated samples, we found 2683 differentially expressed genes (905 upregulated and 1778 downregulated, $\mid \log _{2}$ (fold change) $\left.\mid>1, p_{\text {adj }}<0.005\right)$ (Fig. 3a). We also compared gene expression levels in control or LPAR1-depleted cells after LPA treatment, and found 2532 differentially expressed genes (1540 upregulated and 992 downregulated, $\mid \log _{2}$ (fold change) $\mid>1, p_{\text {adj }}<0.005$ ) (Fig. 3a). Based on these data, we next established a group of genes, which is upregulated in control cells after LPA treatment but is downregulated upon LPAR was depleted (Fig. 3b). Among these 251 genes, gene ontology (GO) classification of biological processes revealed a significant enrichment of several terms related to cell cycle (Fig. 3c), and KEGG signaling pathway analysis also revealed that the differentially expressed genes are predicted to be prominently involved in cell cycle pathways (Fig. 3d), which are consistent with our previous data (Supplementary Fig. 2a). Importantly, we found that Aurora A, a well-known gene involved in cilium disassembly, was significant upregulated by LPA in a LPAR-dependent manner (Fig. 3e). We then validated the mRNA expression of Aurora $A$ and top two differentially expressed genes, STC1 and ODC1, and found that these results were coincident with RNA-Seq (Supplementary Fig. 3a). We also detected that the Aurora A protein levels strongly accumulated at $18-24 \mathrm{~h}$ after serum or LPA stimulation ${ }^{13}$ (Fig. 3f), and this accumulation was greatly reduced in LPAR1-depleted cells (Fig. 3f).

A substantial body of evidence proved that the activation of Aurora A through phosphorylation occurred after serum stimulation, which plays a key role in cilia disassembly ${ }^{13,44,45}$. Next, we explored the effect of LPA on the activation of Aurora A. Consistent with the results of serum stimulation, phosphorylated Aurora A appeared at $2 \mathrm{~h}$ during ciliary disassembly following LPA treatment, and this phosphorylation can be blocked by LPAR1 inhibitor-Ki16425 (Fig. 3g). Given that the level of Aurora A has a little increase at $2 \mathrm{~h}$ after LPA treatment, we speculated that LPA might also be involved in the phosphorylation-mediated activation of Aurora A.

Since Aurora A plays an important role in cilia disassembly, we hypothesized that LPA may regulate cilia disassembly through Aurora A-HDAC6 signaling pathway. To test this hypothesis, we knocked down Aurora A in RPE-1 cells, and found that depletion of Aurora A abolished both serum- and LPA-induced cilia disassembly (Fig. $3 \mathrm{~h}$ and Supplementary Fig. 3b). Histone deacetylase 6-HDAC6 as the downstream of Aurora A is wellknown to deacetylate tubulin for depolymerizing the ciliary axoneme ${ }^{13}$. Next, we found the LPA-induced cilia disassembly was also markedly reduced after tubacin treatment (Supplementary Fig. 3c). Meanwhile, LPA treatment has no influence on the interaction between Aurora A and HDAC6 (Supplementary Fig. 3d). In addition, we also detected the influence on cilia disassembly of top two differentially expressed genes, STC1 and ODC1. The data showed that these two genes have no significant effects on cilia disassembly (Supplementary Fig. 3e-h). Taken together, these data suggest that LPA induces cilia disassembly mainly through Aurora A signaling pathway.

LPA signaling modulates Aurora A to trigger cilia disassembly through YAP/TAZ and calcium/CaM pathway. We next first investigated how LPA regulated the mRNA accumulation of Aurora A. LPA has been shown to inhibit Hippo pathway to activate YAP/TAZ ${ }^{46}$. A separate study has implicated YAP/TAZ in promoting the transcription of Aurora $\mathrm{A}^{47}$. We thus tested whether YAP/TAZ are involved in LPA-induced cilia disassembly. Strikingly, depletion of YAP/TAZ abolished the serumor LPA-induced accumulation of Aurora A proteins and mRNAs (Fig. 4a, b). Consistent with previous studies ${ }^{47}$, both serum and LPA caused a significant nuclear accumulation of YAP in RPE-1 cells (Supplementary Fig. 4a). Furthermore, LPAR1 depletion reduced this nuclear accumulation (Supplementary Fig. 4a). More importantly, cilia disassembly triggered by LPA or serum was blocked by YAP/TAZ co-depletion (Fig. 4c and Supplementary Fig. 4b). These data suggest that LPA in serum acts through YAP/ TAZ to elicit the transcription of Aurora A.

Next, we investigated how LPA regulate the phosphorylation of Aurora A. Since calcium/calmodulin (CaM) are required for Aurora A activation during cilia disassembly ${ }^{44}$ and LPA-LPA receptors can also induce $\mathrm{Ca}^{2+}$ mobilization in diverse cell types ${ }^{48-50}$, we thus detect whether $\mathrm{Ca}^{2+}$ signaling participate in the LPA-induced activation of Aurora A. As expected, we indeed observed the activation of $\mathrm{Ca}^{2+}$ signaling in ciliated RPE-1 cells after LPA treatment (Supplementary Fig. 4c). Importantly, the phosphorylation of Aurora A induced by LPA was blocked by the 
a

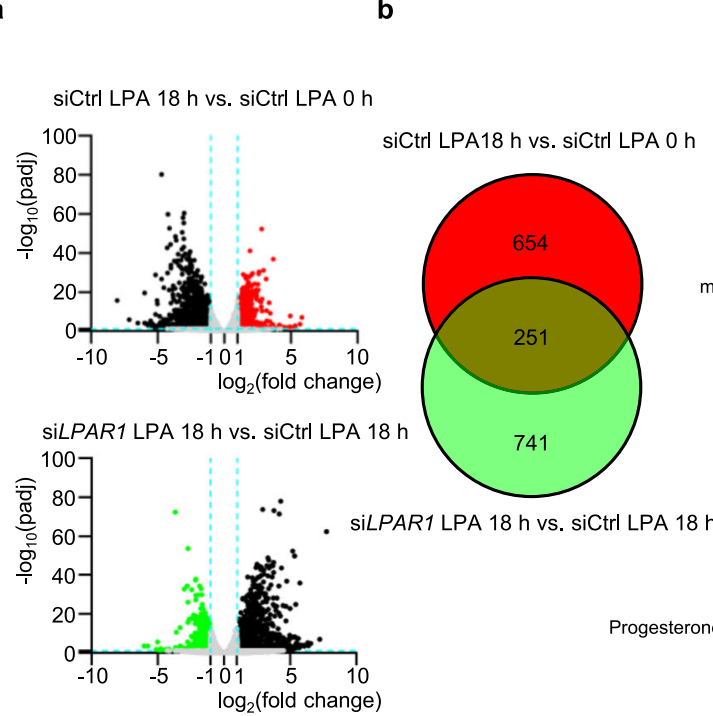

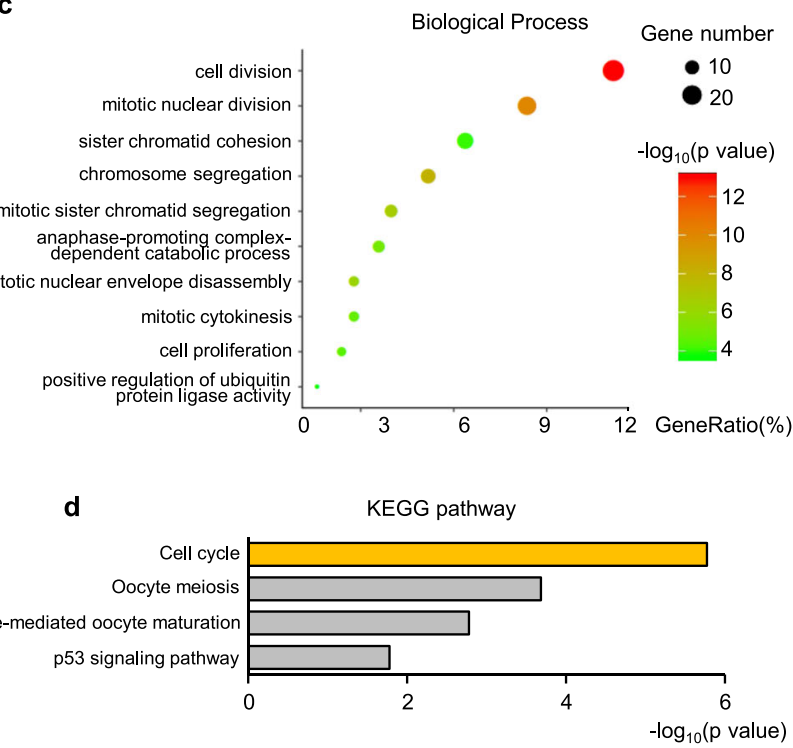

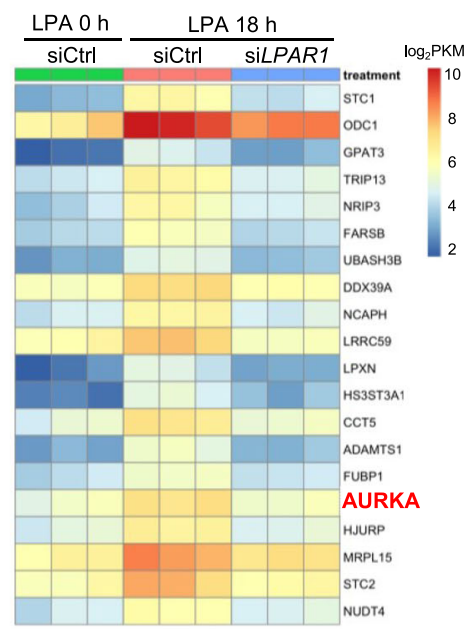

f

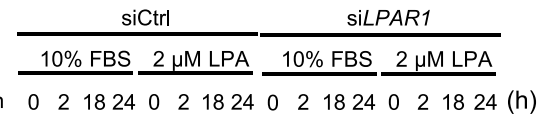

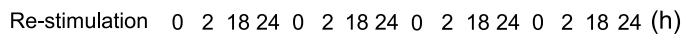

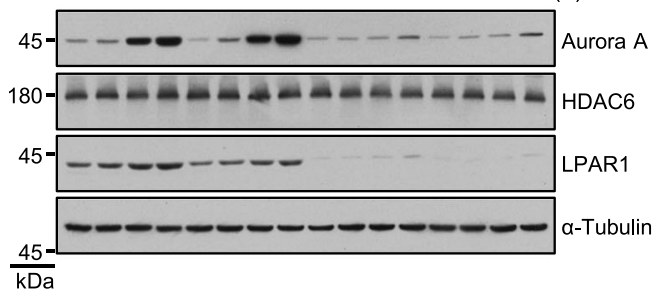

h

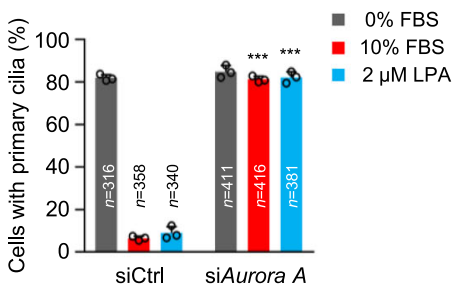

g

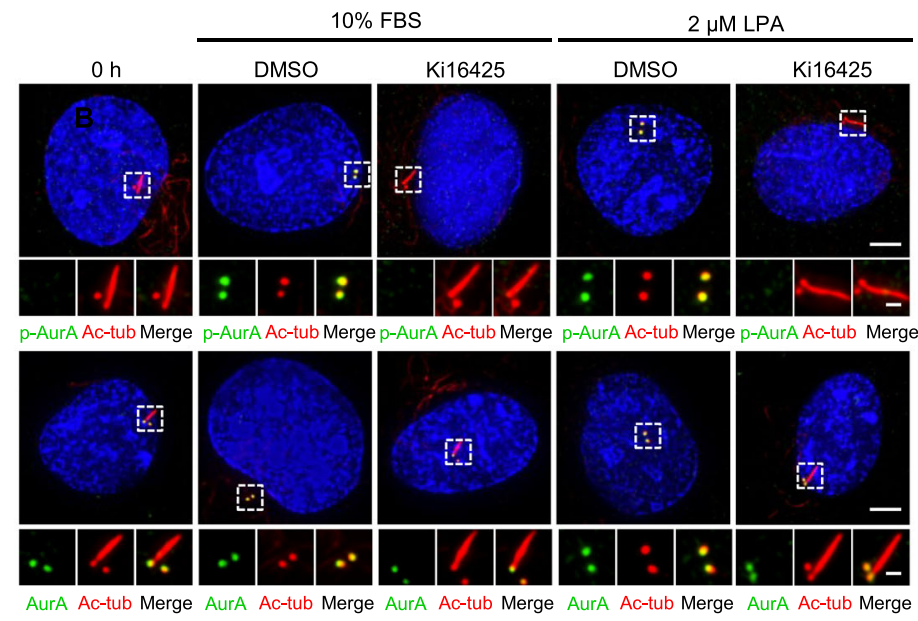

CaM inhibitor calmidazolium (CMZ), the calcium chelator EGTA (Fig. 4d). Importantly, the ability of LPA to induce cilia disassembly was also abolished by CaM inhibitor (Fig. 4e), suggesting that LPA-LPAR1-calcium/CaM was responsible for the phosphorylation of Aurora A and subsequent cilia disassembly. Taken together, these above data suggest that LPA activate Aurora A through calcium pathway and elicit the transcription of Aurora A through YAP/TAZ pathway, thereby initiating cilia disassembly (Fig. 4f).

Lpar1 is required for the generation/maintenance of NPCs and proper neurogenesis. We next explored the physiological role of LPA-induced cilia disassembly in mice. Lpar1 is enriched in 
Fig. 3 LPA signaling initiates cilia disassembly through Aurora A. a Scatter plot analysis of transcriptome expression profiles of siCtrl LPA $18 \mathrm{~h}$ versus siCtrl LPA $0 \mathrm{~h}$, and siLPAR1 LPA $18 \mathrm{~h}$ versus siCtrl LPA $18 \mathrm{~h}$ samples in RPE-1 cells. Red dots and green dots highlight the significantly upregulated or downregulated expressed genes, respectively. b The Venn diagram shows the overlap of upregulated and downregulated genes in a. c Statistics of enriched GO terms display 251 overlapped genes in (b, the brown part). The size of the point indicates the number of differentially expressed genes in this pathway, and the color of the points corresponds to a different p-value range. $\mathbf{d}$ Histogram of the enriched of KEGG pathway of 251 overlapped genes in (b, the brown part). e Heatmaps showing TOP20 enriched genes in (b, the brown part). $\mathbf{f}$ Immunoblot analysis was carried out using indicated antibodies. RPE- 1 cells were starved for $12 \mathrm{~h}$ and then transfected with control siRNA or LPAR1 siRNA. Following serum starvation for another $48 \mathrm{~h}$, cells were treated with $10 \%$ FBS or $2 \mu \mathrm{M}$ LPA for indicated time points. $\mathbf{g}$ LPA activates Aurora A through phosphorylation in RPE-1. Ciliated RPE-1 cells were pretreated with Ki16425 (40 $\mu$ M) or DMSO control for 30 min, and then cells were stimulated with $10 \%$ FBS or $2 \mu \mathrm{M} \mathrm{LPA}$ for $2 \mathrm{~h}$. Cells were stained with anti-p-AurA (Aurora A, green) and anti-Ac-tub (Ac-tubulin, red) antibodies or anti-AurA (Aurora A, green) and anti-Ac-tub (Ac-tubulin, red) antibodies, respectively. Scale bar: $5 \mu \mathrm{m}$ (main image) and $1 \mu \mathrm{m}$ (magnified region). $\mathbf{h}$ The effect of serum- or LPA-induced cilia disassembly in Aurora A knockdown cells. Source data are provided as a Source Data file. Three experiments were repeated independently with similar results in $\mathbf{f}$ and $\mathbf{g}$. Data are presented as mean \pm S.D. of three independent experiments in $\mathbf{h}$. $n$, number of cells. ${ }^{\star \star \star} P<0.001$. Two way ANOVA test was performed followed by Dunnett's multiple comparisons in $\mathbf{h}$.

mouse VZ/SVZ ${ }^{51}$. LPA and its major producing enzyme autotaxin (Atx) are present in cerebrospinal fluid (CSF) in rat $^{52}$. We thus tested whether LPA-LPAR-induced cilia disassembly is involved in VZ/SVZ generation and expansion during mouse cortical development.

We first verified the expression of Lpar1 and Atx in mouse VZ/ SVZ by RNAscope assay. We found that Lpar1 mRNA was also enriched in the cerebral cortical VZ/SVZ niche. Interestingly, Lpar1 expression in VZ/SVZ is developmentally regulated, reaching maximum at El4.5 and declining to very low levels at P0 (Fig. 5a-c). Furthermore, we found that both Lpar1 and Atx were highly expressed in the RG cells of E14.5 mouse cerebral cortex (Supplementary Fig. $4 \mathrm{a}-\mathrm{c}$ ). Given that RG cells is close to the ventricle, we speculated that Atx protein might secrete into the CSF to synthesize LPA, by which Lpar1 is activated in the RG cells.

Previous studies showed that targeted deletion of Lpar1 results in $~ 50 \%$ neonatal lethality, impaired suckling in neonatal pups, and adult Lpar1 knockout survivors showing reduced size and craniofacial dysmorphism ${ }^{32}$. Importantly, both Lpar1 KO and Yap cKO mice showed obvious neurogenesis defects during cerebral cortex development, which was closely related to ciliogenesis $^{53-56}$. We next examined the distribution patterns of NPCs in the developing cortex of Lpar1-knockout mice ${ }^{32}$. We detected radial glia (RG) cells in the VZ with Pax6 and intermediate progenitor cells (IPs) in the SVZ with Tbr2. Compared with the Lpar1 ${ }^{+/+}$control at E14.5 and P0, the total numbers of both Pax6- and Tbr2-positive cells were markedly reduced in the Lpar1 ${ }^{-1-}$ cortex (Fig. 5d-g). These NPCs also failed to expand to upper layers in the Lpar1 $^{-1-}$ cortex at P0 (Fig. 5f). The NPCs from VZ/SVZ produce projection neurons for all layers of cerebral cortex. Decrease in the number of NPCs resulted in a reduction of cortical neuron density in the Lpar1-Icortex at P0 (Fig. 5h, i). Taken together, Lpar1 is required for generation or maintenance of NPCs and proper neurogenesis.

Lpar1 modulates ciliogenesis and neurogenesis during cortical development. Next, we evaluated whether Lpar1 depletion influences the proliferation and self-renewal of NPCs. VZ/SVZ cells in Lpar1 $1^{-1-}$ mice exhibited a lower mitotic index, suggesting that Lpar1 is required for the proliferation of NPCs (Fig. 6a, b). In addition, the apoptosis index was slightly increased in the $L$ par1 $1^{-1-}$ cerebral cortex as compared to Lpar1 $1^{+/+}$controls, which is consistent with previous study ${ }^{53}$ (Supplementary Fig. 5d, e). To test if Lpar1 ${ }^{-1-}$ mice exhibited decreased self-renewal of NPCs, we performed a pulse-chase assay by sequential BrdU and EdU labeling in Lpar $1^{+1+}$ and Lpar $^{-1-}$ mice (Fig. 6c). A smaller percentage of NPCs of Lpar1-/- VZ/SVZ exhibited EdU incorporation, confirming the requirement of Lpar1 in NPC proliferation (Fig. 6c, d). The proportions of self-renewing NPCs $\left(\mathrm{BrdU}^{+} \mathrm{EdU}^{+}\right.$to $\mathrm{BrdU}^{+}$ cells) were significantly decreased in $L p a r 1^{-1-}$ mice as compared to Lpar $1^{+/+}$controls (Fig. 6c, d), indicating that Lpar1 is also required for NPCs self-renewal. Consistent with a requirement of LPAR1 for cilia disassembly, the length of cilia was significantly longer in Lpar $^{-1-}$ RG cells than that in Lpar1 ${ }^{+/+}$cells (Fig. 6e, f and Supplementary Fig. 5f). Moreover, knockdown of the negative regulator of cilia assembly (CP110, Cep97) in RPE-1 cells did not affect the cilium length ${ }^{57}$, but the cilium length was elongated when canonical disassembly factors were depleted ${ }^{14}$, which supports our notion that Lpar1 regulates ciliogenesis potentially through cilia disassembly mechanism.

To further confirm this notion, we isolated NPCs from ppar1 $^{+/+}$ and Lpar $1^{-1-}$ cerebral cortex and performed following experiments with or without overexpressing GFP-Lpar1. We found that overexpression of GFP-Lpar1 robustly rescued the elongated cilia and the reduction of NPCs proliferation in Lpar1-/- NPCs (Fig. 6g, $\mathrm{h}$ and Supplementary Fig. 5g, h). In contrast, the rescued effect of GFP-Lpar1 was destroyed in Lpar1 $1^{-1-}$ NPCs treated with tubacin (a HDAC6 inhibitor) (Fig. 6g, h and Supplementary Fig. 5g, h). Thus, these data suggest that LPA-Lpar1 pathway is essential for the proliferation of NPCs potentially by regulating cilia disassembly.

The transcription levels of the canonical cilia disassembly regulators, Aurora A were dramatically downregulated in RG cells in Lpar $^{-1-}$ mice (Fig. 6i). The mRNA expression of CTGF (a known LPA-inducible and YAP-dependent gene) was also obviously repressed in $L p a r 1^{-1-}$ mice (Fig. 6i). Moreover, we found that the nuclear localization of Yap was also significantly decreased in Lpar1 $^{-/-}$mice compared to Lpar1 $1^{+/+}$controls (Fig. 6j, k), suggesting that the activity of Yap is decreased in Lpar $1^{-I-}$ mice. Thus, these data indicate that LPA-LPAR-YAP pathway is required for the transcription of major cilia disassembly genes in mice.

Taken together, these results indicated that LPA-LPAR pathway is essential for the proliferation of NPCs in VZ/SVZ and subsequent neurogenesis potentially by regulating cilia dynamic and cell cycle progression during mouse embryo development. Further, recent studies reported that the knockout of ATX in the early zebrafish embryo results in defects in left right (L-R) patterning and the Kupffer's vesicle (KV), which further support our findings ${ }^{58,59}$.

\section{Discussion}

Cell-intrinsic regulatory mechanisms of cilia disassembly has been well-studied ${ }^{12,60}$. In contrast, extracellular signals that control cilia disassembly have remained elusive. Using serum as an entry point, our study has identified lysophosphatidic acid (LPA) as a physiological extracellular ligand that promotes cilia disassembly. The LPA pathway acts upstream of canonical disassembly modulators through activating a YAP/TAZ-dependent transcriptional program. Given that many ligands, such as 
a

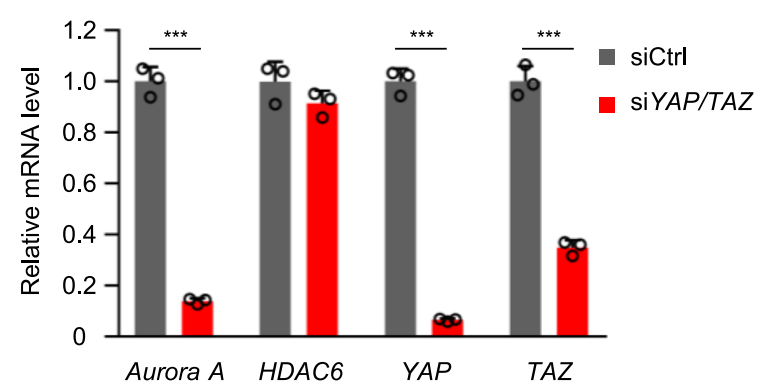

C

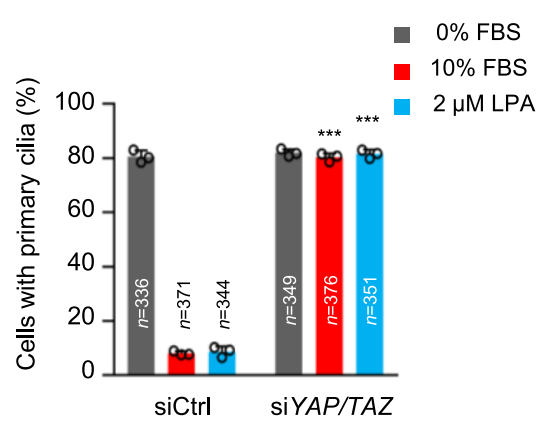

d
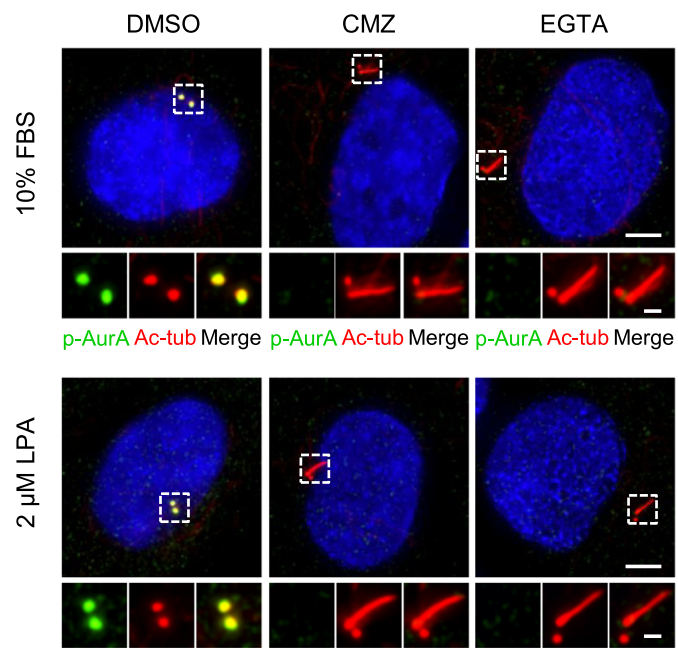

p-AurA Ac-tub Merge p-AurA Ac-tub Merge p-AurA Ac-tub Merge b
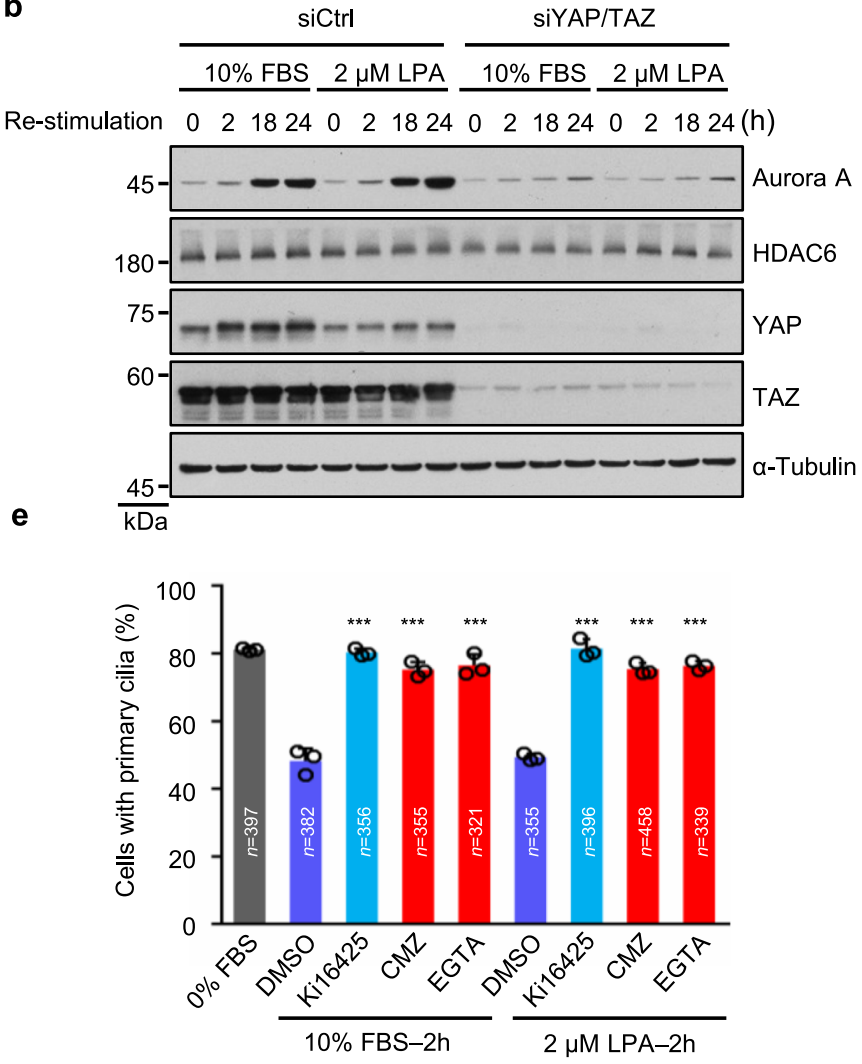

f

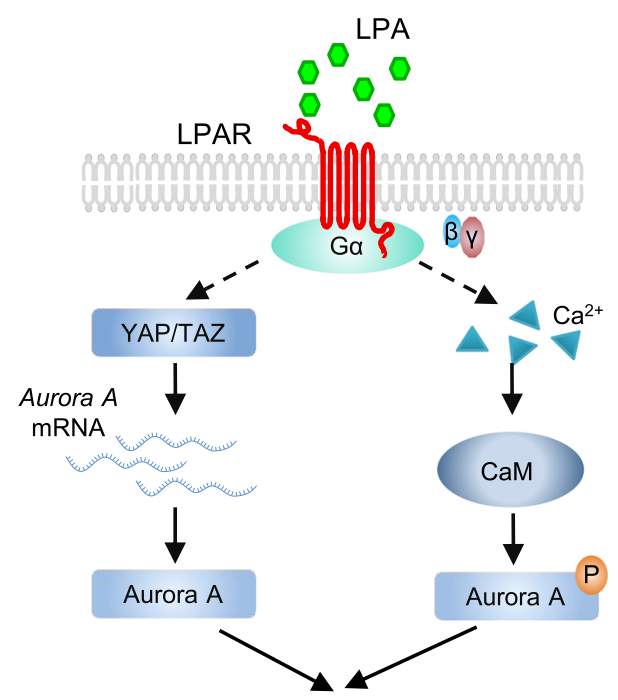

Cilia disassembly

Fig. 4 LPA signaling modulates Aurora A through YAP/TAZ and calcium/CaM pathway. a YAP/TAZ is required for the LPA-induced the transcription of Aurora $\mathrm{A}$. RPE-1 cells were starved for $12 \mathrm{~h}$ and then transfected with control siRNA or YAP/TAZ siRNAs. Following serum starvation for another $48 \mathrm{~h}$, cells were treated with $2 \mu \mathrm{M}$ LPA for $18 \mathrm{~h}$, and the mRNA levels were measured by qPCR. b Immunoblot analysis in control or YAP/TAZ knockdown cells using indicating antibodies. RPE-1 cells were transfected and treated as described in Fig. 3f. c The effect of serum- or LPA-induced cilia disassembly in control or YAP/TAZ knockdown cells. RPE-1 cells were transfected and treated as described in Fig. 2b, c. d CMZ or EGTA blocks serum- and LPA- induced Aurora A activation. Ciliated RPE-1 cells were pretreated with CMZ $(5 \mu \mathrm{M})$, EGTA $(0.5 \mathrm{mM})$ or DMSO control for 30 min, and then cells were stimulated with $10 \%$ FBS or $2 \mu \mathrm{M} \mathrm{LPA}$ for $2 \mathrm{~h}$. Cells were stained with anti-p-AurA (Aurora A, green), anti-Ac-tub (Ac-tubulin, red) antibodies. Scale bar: $5 \mu \mathrm{m}$ (main image) and $1 \mu \mathrm{m}$ (magnified region). e CMZ or EGTA blocks serum- and LPA- induced cilia disassembly. Ciliated RPE-1 cells were pretreated with Ki16425 (40 $\mu \mathrm{M}$ ), CMZ $(5 \mu \mathrm{M})$, EGTA $(0.5 \mathrm{mM})$ or DMSO control for $30 \mathrm{~min}$, and then cells were stimulated with $10 \%$ FBS or $2 \mu \mathrm{M}$ LPA for $2 \mathrm{~h}$. f A proposed model for LPA signaling in the regulation of cilia disassembly. Source data are provided as a Source Data file. Three experiments were repeated independently with similar results in $\mathbf{b}$ and $\mathbf{d}$. Data are presented as mean \pm S.D. of three independent experiments in $\mathbf{a}, \mathbf{c}$, and $\mathbf{e}$. n, number of cells. ${ }^{\star \star \star} P<0.001$. Two-tailed Student's $t$-test in $\mathbf{a}$, two-way ANOVA test was performed followed by Dunnett's multiple comparisons in $\mathbf{c}$ and $\mathbf{e}$. 
b

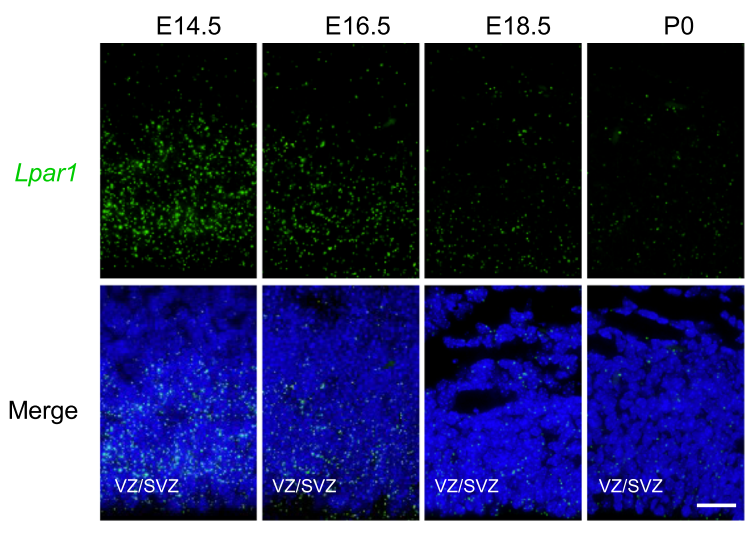

c a

Cortex

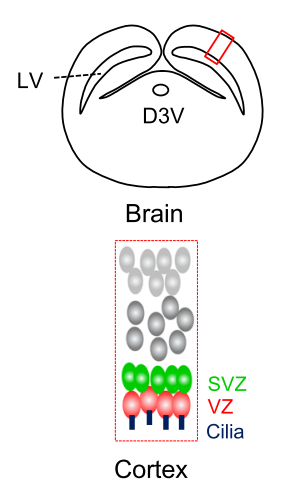

d e $\mathbf{f}$

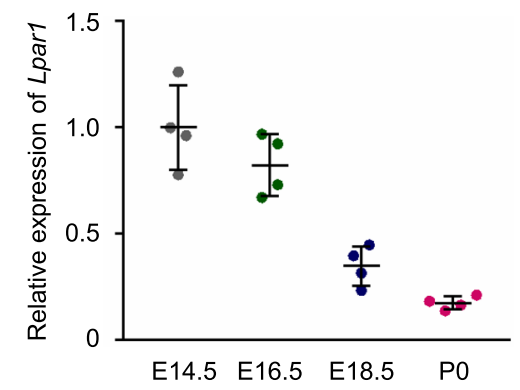

g

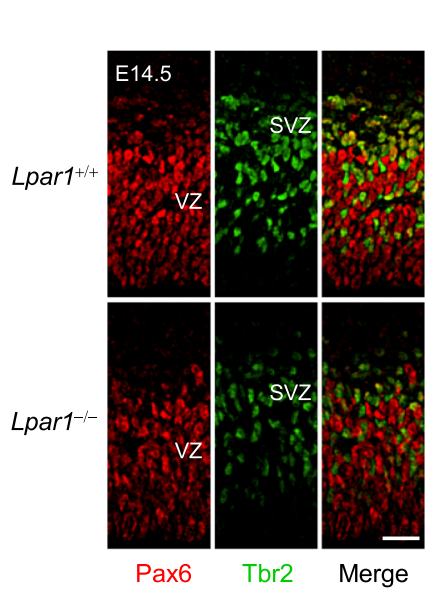

h

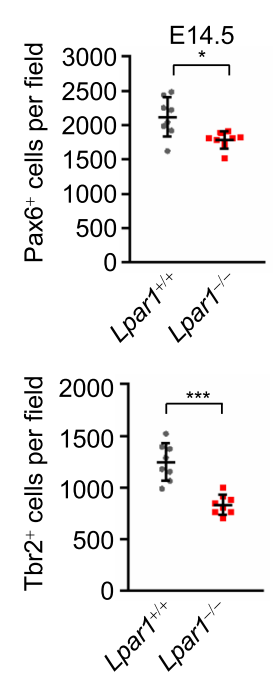

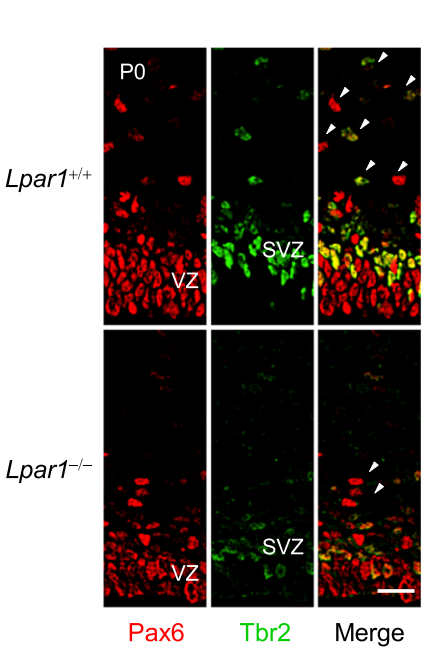
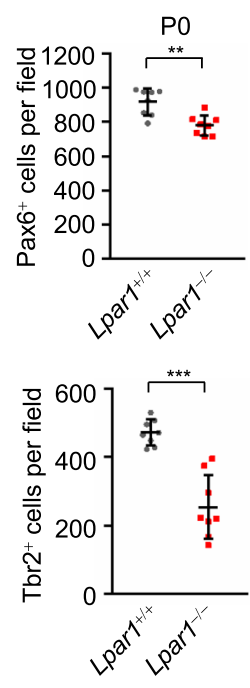

i

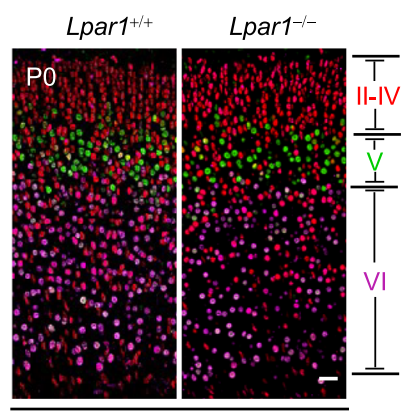

Satb2 + Ctip2 + Tbr1

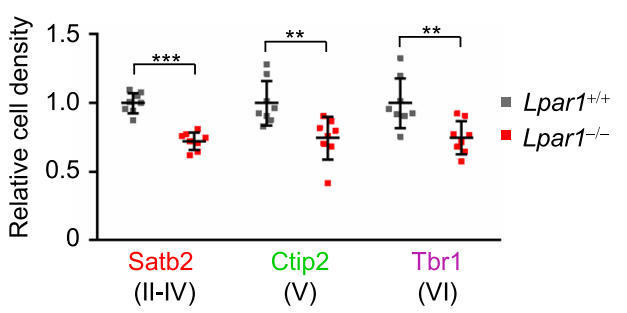

Fig. 5 Lpar1 is required for proper neurogenesis during cortical development. a Schematic diagram of the VZ and SVZ in cerebral cortical. LV, lateral ventricles; D3V, dorsal 3rd ventricle; VZ, ventricular zone; SVZ, subventricular zone. b Expression of Lpar1 in mouse cortex. RNAscope fluorescent in situ hybridization of Lpar1 in cortex with wide-type mice at E14.5, E16.5, E18.5 and P0, Lpar1 mRNA puncta (green), DNA (blue). Scale bar, $20 \mu$ m. c Quantification of Lpar1 hybridization signals in $\mathbf{b}, n=4$ mice. $\mathbf{d}-\mathbf{g}$ At E14.5 and PO, the total numbers of both Pax6 $6^{+}$and Tbr2+ cells are reduced in the $\mathrm{Lpar1}^{-/-}$than in $L$ par1 ${ }^{+/+}$mice cortex. d, f Representative images of $L$ par1 ${ }^{+/+}$and $L$ par1 ${ }^{-/-}$mice cortices at E14.5 (d) and PO (f), which were stained with antibodies against Pax6 (red, radial glia cells marker) and Tbr2 (green, intermediate progenitor cells marker). White arrows indicate the neural progenitor cells (NPCs) expanded to the upper layers of cortex (f). Scale bar, $20 \mu \mathrm{m}$. e, g Quantification of Pax6 ${ }^{+}$cells and Tbr2+ cells at E14.5 (e) and P0 (g) cortices respectively, $n=8$ sections from four mice. $\mathbf{h} L p a r 1^{-/-}$mice exhibited a reduction of cortical neuron density at PO. $L p a r 1^{+/+}$and $L p a r 1^{-/-}$cortices in mice were stained with layer II-IV marker (Satb2, red), layer V marker (Ctip2, green) and layer VI marker (Tbr1, purple). Scale bar, $20 \mu \mathrm{m}$. i Quantification of the relative cell density in $\mathbf{h}, n=8$ sections from four mice. Source data are provided as a Source Data file. Four experiments were repeated independently with similar results in $\mathbf{b}, \mathbf{d}, \mathbf{f}$ and $\mathbf{h}$. Data are presented as mean \pm S.D. in $\mathbf{c}, \mathbf{e}, \mathbf{g}$, and $\mathbf{i} .{ }^{\star} P<0.05,{ }^{\star \star} P<0.01,{ }^{\star \star \star} P<0.001$. Two-tailed Student's $t$-test. 

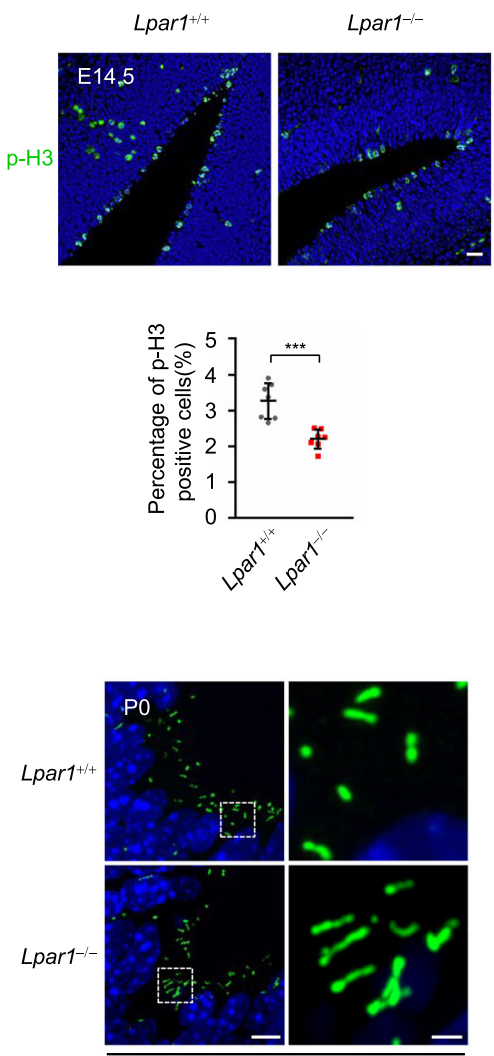

ARL13B c

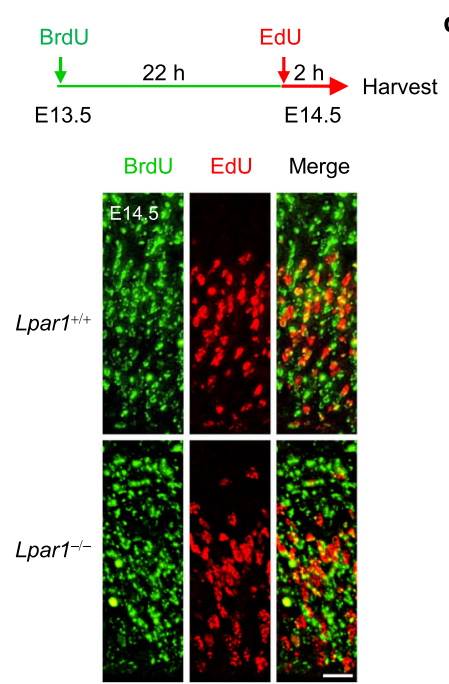

f

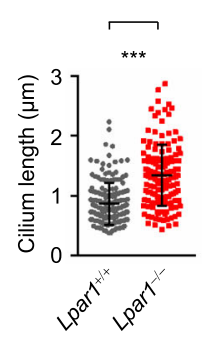

i
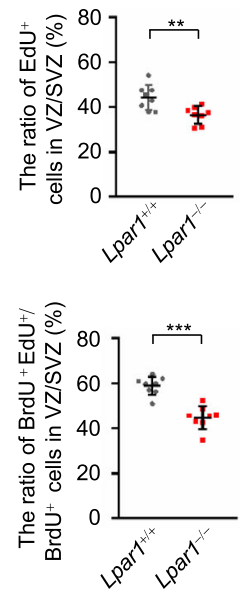

g

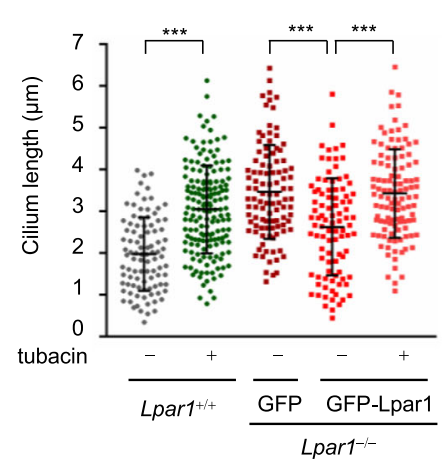

h

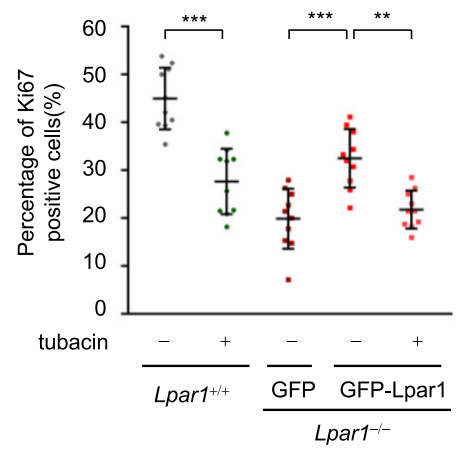

estrogen, secretin and platelet-activating factor, can active YAP/ TAZ acting through their receptor GPCRs ${ }^{46}$, it is likely that these GPCRs could similarly modulate ciliogenesis as LPA receptors. Future studies are needed to address these important issues under specific physiological and pathological contexts.

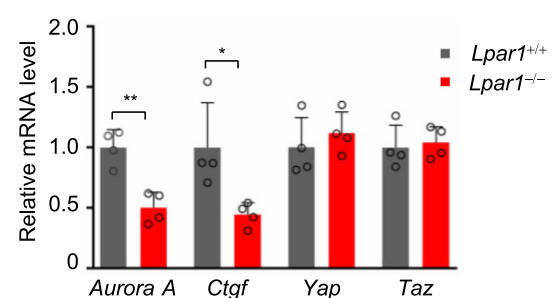

j

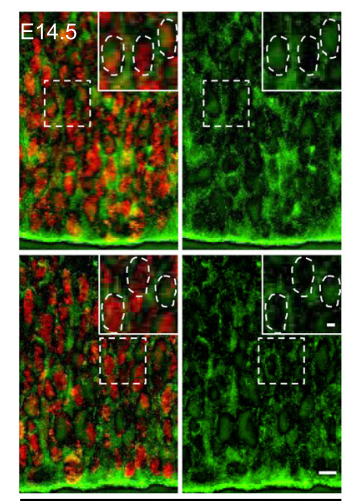

Pax6 + YAP

$\mathbf{k}$

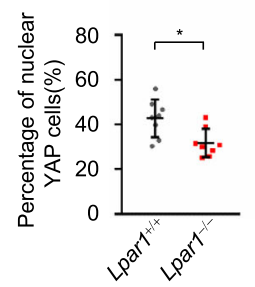

Activation of Aurora A kinase have been well-studied in cilia disassembly $13,16,44,61,62$, whereas the levels are also dramatically upregulated during cilia disassembly. Thus far, the mechanisms governing the dynamic regulation of Aurora A levels during the cilia life cycle remain unclear. Our study provides the explanation 
Fig. 6 NPCs of Lpar1 ${ }^{-1-}$ mice exhibit elongated cilia and decreased cell division. $\mathbf{a}, \mathbf{b} \vee V Z / S V Z$ cells in $L$ par1 ${ }^{-/-}$mice exhibit a lower mitotic index. a Representative images of mitotic cells stained with phosphorylated histone 3 (p-H3, green) and DNA (blue) in E14.5 Lpar1 ${ }^{+/+}$and Lpar1 ${ }^{-/-}$mice cortices. Scale bar, $20 \mu \mathrm{m}$. b The percentage of $\mathrm{p}-\mathrm{H} 3$-positive cells from VZ/SVZ cells in $\mathbf{a}, n=8$ sections from four mice. c Representative images of E14.5 Lpar1 $+/+$ and Lpar1 $^{-/}$- cortices subjected to dual pulse-chase labeling of BrdU (green) and EdU (red). The pulse-chase timing is shown on the top. Scale bar, $20 \mu \mathrm{m}$. d Quantification of the ratio of EdU + cells in VZ/SVZ (top) and the ratio of BrdU $+\mathrm{EdU}^{+}$to $\mathrm{BrdU}^{+}$cells in VZ/SVZ (down), $n=8$ sections from four mice. e Lpar1 $^{-/-}$RG cells possess longer cilia than $L$ par1 ${ }^{+/+}$. Lpar1 ${ }^{+/+}$and $L$ par1 ${ }^{-/-}$cortices section at PO were stained with cilia marker (ARL13B, green) and DNA (blue). Scale bar, $5 \mu \mathrm{m}$ (main image) and $1 \mu \mathrm{m}$ (magnified region). $\mathbf{f}$ Quantification of the cilium length in e, $L$ par $1^{+/+}: n=179$ cilia from four mice, Lpar1 ${ }^{-/-}: n=149$ cilia from four mice. $\mathbf{g}$, $\mathbf{h}$ Quantification of cilium length $(\mathbf{g})$ or percentage of Ki67+ cells (h) in Sox2-positive RG cells. Isolated cells from Lpar ${ }^{+/+}$cerebral cortex were treated with $2 \mu \mathrm{M}$ tubacin or DMSO for $72 \mathrm{~h}$, and isolated cells from Lpar ${ }^{-/-}$cerebral cortex were infected with lentivirus expressed GFP or GFP-Lpar1 with or without $2 \mu \mathrm{M}$ tubacin treatment for $72 \mathrm{~h}$. $\mathbf{g}$ From left to right, $n=95,164,103$, 111 cilia from four experiments. $\mathbf{h} n=10$ mice from four experiments. i qPCR analyzed the transcriptional levels of Aurora A, Ctgf, Yap and Taz in E14.5 Lpar1 $7^{+/+}$and Lpar1 ${ }^{-/-}$mice cortices. $\mathbf{j}$ Representative images of E14.5 $\mathrm{Lpar1}^{+/+}$and $\mathrm{Lpar}^{-/-}$cortices stained with YAP (green) and PAX6 (red). Scale bars, $20 \mu \mathrm{m}$ (main image) and $5 \mu \mathrm{m}$ (magnified region). $\mathbf{k}$ Quantification of the percentage of PAX6 $6^{+}$cells with nuclear YAP in VZ. Source data are provided as a Source Data file. Four experiments were repeated independently with similar results in $\mathbf{a}, \mathbf{c}, \mathbf{e}$, and $\mathbf{j}$. Data are presented as mean \pm S.D. in $\mathbf{b}, \mathbf{d}, \mathbf{f}, \mathbf{g}, \mathbf{h}, \mathbf{i}$, and $\mathbf{k}$. ${ }^{\star} P<0.05$, ${ }^{\star \star} P<0.01,{ }^{\star \star \star} P<0.001$. Two-tailed Student's $t$-test in $\mathbf{b}, \mathbf{d}, \mathbf{f}, \mathbf{i}$, and $\mathbf{k}$; One-way ANOVA test was performed followed by Bonferroni's multiple comparisons in $\mathbf{g}$ and $\mathbf{h}$.

that the accumulation of Aurora A results from the increase of its transcription by LPA pathway-mediated activation of YAP/TAZ.

By systematic separation of components, we discovered LPA as the major factor in serum for driving cilia disassembly through Aurora A, while the recent work of Walia et al. identified a negative role for LPA in cilia assembly through Rab8/ Rab $11^{43}$. Actually, it is generally accepted that the assembly and disassembly of cilia are dynamically balanced processes. Previous study showed that depletion of canonical disassembly factors, including Nek2 and Kif24, could directly induce ciliogenesis in serum-cultured cells ${ }^{14}$. We also observed that knockdown of LPAR1 or Aurora A directly resulted in cilia formation in serum-cultured cells. Therefore, the disruption of disassembly can directly release the assembly activity and trigger the early events of cilium biogenesis even in the presence of serum, just as observed by Walia. Our findings together with Walia's work will contribute to the comprehensive understanding about the extracellular signal controlling ciliogenesis, and LPA is expected to play important roles in many specific physiological and pathological contexts through regulating proper ciliogenesis.

NPCs in VZ/SVZ niche undergo both asymmetric and symmetric cell divisions for self-renewal and differentiation ${ }^{29}$. These divisions are regulated by developmental cues and extracellular signals. The primary cilia of these NPCs can detect diverse signaling factors in cerebrospinal fluid (CSF), such as sonic hedgehog (shh) and $\mathrm{IGF}^{63,64}$. On the other hand, the mitotic entry of NPCs requires the disassembly of their primary cilia ${ }^{26-28}$. Inactivation of cell-intrinsic cilia disassembly factors has been reported to cause abnormally elongated cilia and limit the proliferation of NPCs in $\mathrm{VZ}^{65}$. However, the extracellular factors that control cilia disassembly during cortical development are unknown. We show that LPA not only promotes cilia disassembly in cultured cells, but also regulates proper ciliogenesis during mammalian development. Our results now establish LPA as one such physiological extracellular factor in this process.

We propose that LPA in the CSF inhibits ciliogenesis through promoting cilia disassembly. However, the mouse embryo is so small that it is technically unfeasible to directly detect the presence of LPA in CSF during embryo development. We found that the Atx mRNA was highly expressed in the RG cells of E14.5 mouse VZ region, which is close to the ventricle (Supplementary Fig. 5a). Thus, we propose that as an exoenzyme, Atx protein is likely secreted into CSF to synthesize LPA in CSF, and this issue will be further analyzed in future studies.

Loss of LPA signaling through genetic ablation of LPAR1 causes elongated cilia and defective NPC proliferation and self- renewal. Interestingly, LPAR1 expression is enriched in NPCs and is developmentally regulated. Its expression level positively correlates with NPC proliferation. Its expression is high at E14.5 when NPCs undergo fast proliferation. Its expression is very low at P0 when NPC proliferation slows down. Therefore, we have discovered a developmentally regulated extracellular signaling that coordinates ciliogenesis and neurogenesis. All of the above results provide a different view into cilia disassembly during embryonic development and ciliopathy.

LPA production and LPARs expression are both elevated in many types of human cancers ${ }^{66-70}$. Elevated LPA and activation of LPARs have been shown to promote tumorigenesis in mouse models $^{38}$. It is possible that defective ciliogenesis as a result of hyperactive LPA signaling is a causal factor of tumorigenesis. Targeting the LPA pathway with chemical inhibitors might be a potential strategy to counteract the ciliary defect caused by LPA and restrict the proliferation of cancer cells.

\section{Methods}

Mice. The generation and genotyping of Lpar1-deficient mice was described previously ${ }^{32}$. All animal procedures were approved by the Institutional Animal Care and Use. Animals were kept under specific-pathogen-free conditions, with controlled temperature $\left(20-25^{\circ} \mathrm{C}\right)$, humidity $(40-60 \%)$ and light cycle $(12 \mathrm{~h}$ light/ dark). All embryos used for this study were obtained from natural mattings of virgin females 8-12 weeks old. Noon on the day of the discovery of a vaginal plug was considered to be embryonic day E0.5. P0 embryos were collected before the death of $L$ par1 ${ }^{-I-}$ pubs. All animal experiments were performed with the approval of the Institutional Animal Care and Use Committee of Military Medical Sciences (IACUC-DWZX-2019-506).

Cell culture. All cell lines were maintained at $37^{\circ} \mathrm{C}$ with $5 \% \mathrm{CO}_{2}$. hTERT RPE(RPE-1) and IMCD-3 cells were kindly provided by Dr. Xueliang Zhu. HEK293T cells were obtained from the ATCC (CRL-3216). RPE-1 cells were cultured in DMEM/F12 (1:1) supplemented with $10 \% \mathrm{FBS}, 0.01 \mathrm{mg} / \mathrm{mL}$ hygromycin $\mathrm{B}$ and $1 \%$ penicillin/ streptomycin, and IMCD-3 cells were cultured in DMEM/F-12 (1:1) supplemented with $10 \% \mathrm{FBS}$ and $1 \%$ penicillin/streptomycin. HEK293T cells were cultured in DMEM supplemented with $10 \%$ FBS and $1 \%$ penicillin/streptomycin. Primary MEF cells were isolated from E14 wild C57BL/6J mouse embryos using standard protocol and incubated in DMEM medium supplemented with 15\% FBS and $1 \%$ penicillin/ streptomycin. For cilia formation, cells were incubated in DMEM/F12 (1:1) for $48 \mathrm{~h}$; for cilia disassembly, ciliated cells were re-stimulated with DMEM/F12 (1:1) contained with serum, LPA or other reagents as indicated.

Immunofluorescence staining and imaging. To detect primary cilia, Aurora A and p-Aurora A, cells were placed on ice for $10 \mathrm{~min}$, and then fixed and permeabilized in cold methanol for $5 \mathrm{~min}$. Cells transfected with plasmids were fixed in cold methanol for $30 \mathrm{~s}$. To visualize the localization of YAP and detect primary cilia and Ki67 in isolated NPCs, cells were fixed with $4 \%$ paraformaldehyde (PFA) for $10 \mathrm{~min}$ and permeabilized with $0.1 \%$ Triton X-100 in PBS.

For tissue immunofluorescence, brains were fixed in $4 \%$ PFA at $4{ }^{\circ} \mathrm{C}$ overnight and then embedded in paraffin (Thermo Fisher Scientific) or OCT compound 
(Sakura). Paraffin-embedded sections were serially cut at $3 \mu \mathrm{m}$. Frozen sections were cut at $8 \mu \mathrm{m}$.

Cells and tissue sections were blocked with 3\% BSA in PBS for $1 \mathrm{~h}$ prior to incubation with primary antibodies overnight at $4{ }^{\circ} \mathrm{C}$. Secondary antibodies used were Alexa Fluor 488-, 546- and 647-conjugated goat anti-mouse, anti-rabbit, antirat or donkey anti-goat IgG (Thermo Fisher Scientific). To detect the overexpression of exogenous plasmid, cells were stained with ANTI-FLAG ${ }^{\circledR}$ M2FITC antibody for $1 \mathrm{~h}$ at room temperature. DNA was stained with Hoechst 33342 (1:500, H3570, Thermo Fisher Scientific) for $10 \mathrm{~min}$ at room temperature. Slides were then washed three times and mounted. EdU staining was performed using the Cell-Light EdU Apollo567 In Vitro Kit in accordance with the manufacturer's instruction (C10310-1, RIBOBIO). $\mathrm{Ca}^{2+}$ signaling was detected using the Fluo-4, AM in accordance with the manufacturer's instruction (F8500, Solarbio).

Immunofluorescence was detected using 60×/1.42 Oil objective (Figs. 1-4, Supplementary Figs. 1-4) or 20×/0.75 objective (Figs. 5, and 6; and Supplementary Fig. 5) with a DeltaVision (DV) Image Restoration Microscope, a 63×/1.40 oil objective on Zeiss LSM 880 (Fig 6, and Supplementary Fig. 5) or a 63×/1.40 Oil objective on Zeiss LSM 880 with airyscan (Fig. 6 and Supplementary Fig. 5). For data acquisition on a DV Image Restoration Microscope, we used GE healthcare SoftWoRx 6.5.2. For data collection on Zeiss LSM 880 microscope, we used ZEN 2.1 SP2 Black version 13.0.2.518 (ZEISS). All acquisition settings were kept constant for experimental and control groups in the same experiment. The representative images acquired by DV system were processed by iterative constrained deconvolution (SoftWoRx, Applied Precision Instruments). All raw images were analyzed with Volocity 6.0 software (Perkin Elmer). Cilium length was measured from the tip to the base.

Antibodies. Antibodies used in this study included mouse anti-Ac-tubulin antibody (1:800, T6793, Sigma), rabbit anti- $\gamma$-Tubulin antibody (1:600, T6557, Sigma), mouse anti-LPAR1 antibody (1:100, sc-515665, Santa), mouse anti- $\alpha$-Tubulin (1:5000, T5168, Sigma), mouse anti-Flag antibody (1:1000, F3165, Sigma), mouse anti-Ga 12 antibody (1:200, sc-515445, Santa), mouse anti-Ga 13 antibody (1:200, sc-293424, Santa), mouse anti-Ga q antibody (1:200, sc-136181, Santa), mouse anti-Ga 11 antibody (1:200, sc-390382, Santa), mouse anti-YAP antibody (1:1000, sc-101199, Santa), rabbit anti-TAZ antibody (1:4000, 66500-1-Ig, Proteintech), rabbit antiAurora A antibody (1:1000, 4718s, Cell Signaling Technologies), rabbit anti-HDAC6 antibody (1:1000, 7558T, Cell Signaling Technologies), rabbit anti-ARL13B antibody (1:400, 17711-1-AP, Proteintech), mouse anti-BrdU antibody (1:250, 11-286C100, Exbio), rabbit anti-Pax6 antibody (1:500, PRB-278P, Covance), rat anti-Tbr2 antibody (1:500, 14-4875-82, Thermo Fisher Scientific), rabbit anti-Satb2 antibody (1:500, ab92446, Abcam), mouse anti-Tbrl antibody (1:250, 66564-1-Ig, Proteintech), rabbit anti-Ctip2 antibody (1:250, ab28448, Abcam), rabbit anti-p-H3 (Ser10) antibody (1:500, 9701s, Cell Signaling Technologies), rabbit anti-PhosphoAurora A (Thr288) antibody (1:100, MA5-14904, Thermo Fisher Scientific), goat anti-Sox 2 antibody (1:300, Sc-17320, Santa), rabbit anti-Ki67 antibody (1:500, 9129T, Cell Signaling Technologies), rabbit anti-cleaved Caspase3 antibody (1:400, 9664, Cell Signaling Technologies), mouse anti-Flag-M2 FITC (1:8000, F4049, Sigma), Goat anti-Mouse Alexa Fluor 488 (1:500, A11029, Thermo Fisher Scientific), Goat anti-Mouse Alexa Fluor 546 (1:500, A11030, Thermo Fisher Scientific), Goat anti-Mouse Alexa Fluor 647 (1:500, A21235, Thermo Fisher Scientific, lot: 2088736), Goat anti-Rabbit Alexa Fluor 488 (1:500, A11034, Thermo Fisher Scientific), Goat anti-Rabbit Alexa Fluor 546 (1:500, A11035, Thermo Fisher Scientific), Goat anti-Rabbit Alexa Fluor 647 (1:500, A21245, Thermo Fisher Scientific), Goat anti-Rat Alexa Fluor 555 (1:500, A21434, Thermo Fisher Scientific), Goat antiRat Alexa Fluor 488 (1:500, A11006, Thermo Fisher Scientific), Donkey anti-Goat Alexa Fluor 647 (1:500, A21447, Thermo Fisher Scientific).

Chemicals. LPA (18:1, L7260), phosphatidylserine (PS, P7769-5MG) and phosphatidylcholine (PC, P3556-25MG) were purchased from Sigma and all other lipid chemicals were purchased from Avanti Polar Lipids. Other Lipids shown in Fig. 1D and Fig. S1D are lyso phosphatidylserine (LPS, 860081), lyso-phosphatidylcholine (LPC, 850092P), phosphatidylethanolamine (PE, 840465P), lyso phosphatidylethanolamine (LPE, 858127c), phosphatidylglycerol (PG), lyso phosphatidylglycerol (LPG, 830071), phosphatidic acid (PA, 840861P), sphingomyelin (SPH, 860062P), phosphatidylinositol (PI, 850142P), phosphatidylinositol 3-phosphate $\left(\mathrm{PI}_{3} \mathrm{P}, 850150 \mathrm{P}\right)$, phosphatidylinositol(3,4)-bisphosphate $\left(\mathrm{PI}_{3,4} \mathrm{P}, 850153 \mathrm{P}\right)$, cardi olipin (CL, 710335P), 16:0 LPA (857123P), 18:0 LPA (857125c) and 20:4 LPA (857128p)

The LPAR1/3 inhibitor Ki16425 $(40 \mu \mathrm{M}$, S1315, Selleck), HDAC6 inhibitor tubacin $(2 \mu \mathrm{M}, \mathrm{HY}-13428, \mathrm{MCE})$, CaM inhibitor CMZ (5 $\mu \mathrm{M}, 2561, \mathrm{R} \& \mathrm{D})$, calcium chelator EGTA (0.5 mM, HY-D0973, MCE) or DMSO vehicle were added to RPE-1 cells 30 min prior to the treatment of serum or LPA.

RNA extraction, reverse transcription, and real-time PCR. Following various treatments, cells or mouse cerebral cortex were washed with cold phosphatebuffered saline and subjected to RNA extraction using TRIZOL (93289, Sigma). RNA samples were reverse-transcribed to complementary DNA (cDNA) using the PrimeScript $^{\mathrm{TM}}$ RT Master Mix (RR036A, Takara), cDNA was then diluted and used for quantification (with GAPDH gene as a control) by real-time PCR, which was performed on an Roche StepOnePlus Real-Time PCR System and analyzed using the $2^{-\Delta \Delta \mathrm{Ct}}$ method. Primers pairs used in this study are: LPAR1 (Gene ID 1902), GTGTGGGCTGGAACTGTAT CTG/TAGTCCTCTGGCGAACATAG; LPAR2 (Gene ID 9170), GGCCAGTGCTACTACAACGAGACC/TGGAGGCGATGGCT GCTATG AC; LPAR3 (Gene ID 23566), CCTGGTGGTTCTGCTCCTCGAC/GTG CCATACAT GTCCTCGTCCTTG; LPAR4 (Gene ID 2846), ATTGAAGTTGTTG GGTTTATCAT/G CACAAGGTGATTGGGTACAT; LPAR5 (Gene ID 57121) CCTGGCGGCGGTGGT CTACTCGTC/GACCGCCAGCGTGCTGTTGTAGGG; LPAR6 (Gene ID 10161), TTGTATGGGTGCATGTTCAGC/GCCAATTCCGTGT TGTGAAGT; Aurora A (Gene ID 6790), GAGGTCCAAAACGTGTTCTCG/ACA GGATGAGGTACACTGG TTG; STC1(Gene ID 6781), GGCGACCACCAAAGTC AAAC/TACTTGTCGCAT TGGGGTCC; ODC1(Gene ID 4953) GGGCGCTCTG AGATTGTCAC/GGCAATC CGCAAAACCAACT; HDAC6 (Gene ID 10013), AAGAAGACCTAATCGTGGGAC T/GCTGTGAACCAACATCAGCTC; YAP1 (Gene ID 10413), TAGCCCTGCGTAGC CAGTTA/TCATGCTTAGTCCACTGT CTGT; TAZ (Gene ID 25937), GATCCTGCC GGAGTCTTTCTT/CACGTCGTA GGACTGCTGG; GAPDH (Gene ID 2597), GGA GCGAGATCCCTCCAAAAT/ GGCTGTTGTCATACTTCTCATGG; Aurora A (Gene ID 20878), CTGGATGCT GCAAACGGATAG/CGAAGGGAACAGTGGTCTTAAC A; Ctgf (Gene ID 14219), GGGCCTCTTCTGCGATTTC/ATCCAGGCAAGTGCAT TGGTA; Yap1 (Gene ID 22601), TACTGATGCAGGTACTGCGG/TCAGGGATC TCAAAGGA GGAC; Taz (Gene ID 97064), GAAGGTGATGAATCAGCCTCTG/G TTCTGA GTCGGGTGGTTCTG; Gapdh (Gene ID 14433), AGGTCGGTGTGAACG GAT TTG/TGTAGACCATGTAGTTGAGGTCA.

Cloning and plasmids. The resistant plasmid expressing recombinant pCDNA3.0 Flag-LPAR1 was synthesized BioMed Company. The plasmids Ga 12 QL, Ga 13 QL, Ga q QL, Ga i QL, Ga o QL were obtained from Dr. Faxing Yu, and the cDNA of Ga 11 was obtained from Dr. Jiahuai Han. Flag- Ga 12 QL, Flag-Ga 13 QL, FlagGa q QL, Flag-Ga i QL, Flag-Ga o QL were amplified by PCR and cloned into pCDNA3.0, and pCDNA3.0-Flag-Ga 11 Q209L mutant were generated by PCRbased site-directed mutagenesis. All constructs were verified by DNA sequencing Plasmid transfection into RPE-1 cells was performed using Lipofectamine ${ }^{\mathrm{Tx}}$ LTX Reagent (Thermo Fisher Scientific) according to the manufacturer's instruction. Lentivirus expressing GFP or GFP-Lpar1 was purchased from Taitool Bioscience

RNAi. Synthetic small interfering RNA oligonucleotides were synthesized by Sigma or Life Technologies. Transfection of siRNAs using RNAiMAX (Thermo Fisher Scientific) was performed according to the manufacturer's instructions. The sequences of siRNAs are as follows. control siRNA: $5^{\prime}$-UUCUCCGAACGUGUCA CGUTT-3'; LPAR1 siRNA (1\#, sigma): 5'-GAAAUGAGCGCCACCUUUAdTdT' LPAR1 siRNA (1\#, Thermo Fisher Scientific): 5'-CAUCUGCUGGACUCCUGGA UUGGUU-3'; G $\alpha 12$ siRNA (1\#, sigma): 5'-GAGCAUGACUUCGUUAUUAdT dT-3'; G $\alpha 12$ siRNA (2\#, sigma): 5'-CGGUGAAGUACUUCCUGGAdTdT-3'; G $\alpha$ 13 siRNA (1\#, sigma): 5'-CU AUGACCGGCGUCGAGAAdTdT-3'; G $\alpha 13$ siRNA (2\#, sigma): 5'-GUAUGAGGGU GCUGGUUGAdTdT-3'; G $\alpha$ q siRNA (1\#, sigma): 5'-CUCAAGAUCCCAUACAAGU dTdT-3'; G $\alpha$ q siRNA (2\#, sigma): 5'-CAAU AAGGCUCAUGCACAAdTdT-3'; G $\alpha 11$ siRNA (1\#, sigma): 5'-GAGUUCAUCC UGAAGAUGUdTdT-3'; G $\alpha 11$ siRNA (2\#, sigma): 5'-CCAAGCUCGUCUACC AGAAdTdT-3'; STC1 siRNA(sigma): 5'-CCAAC AGAUACUAUAACAGdTdT-3'; ODC1 siRNA(sigma): 5'-GAGATCACCGGCGTA ATCAdTdT-3'; YAP1 siRNA (Thermo Fisher Scientific): 5'-CAGCAGAAUAU GAUGAACUCGGCUU-3'; TAZ (WWTR1) siRNA (Thermo Fisher Scientific): 5'-GAGAAAGGAUUCGAAUGCG CCAAGA-3'; Aurora A siRNA (Thermo Fisher Scientific): 5'- GGGUAAAGGAA AGUUUGGUAA UGUU-3'.

Immunoprecipitation. For immunoprecipitation (IP) experiments, indicated plasmids were transfected into HEK293T cells. At $6 \mathrm{~h}$ post-transfection, cells were treated with $40 \mu \mathrm{M}$ Ki16425 or DMSO for $24 \mathrm{~h}$. Cells were then lysed in M2 buffer (50 mM Tris-HCl, pH 7.5, 1\% NP-40, $150 \mathrm{mM} \mathrm{NaCl}, 0.5 \mathrm{mM}$ EGTA, $0.5 \mathrm{mM}$ EDTA, $1 \mathrm{mM}$ DTT, $1.5 \mathrm{mM} \mathrm{MgCl}_{2}, 1 \mathrm{mM}$ PMSF), and incubated with anti-Flag M2 affinity gel (A2220, Sigma) for $4 \mathrm{~h}$ at $4{ }^{\circ} \mathrm{C}$. The immunoprecipitated proteins were immunoblotted with indicated antibodies.

Western blot. Cell lysates were prepared in M2 buffer $(20 \mathrm{mM}$ Tris-HCl, pH 7.5 $250 \mathrm{mM} \mathrm{NaCl}, 3 \mathrm{mM}$ EDTA, $3 \mathrm{mM}$ EGTA, 0.1\% NP-40) with complete protease inhibitor cocktail (Roche). Protein isolated from cell lysates was quantified with the Bradford assay (Biorad). Protein was boiled at $95^{\circ} \mathrm{C}$ for $5 \mathrm{~min}$ prior to being loaded into a $4-12 \%$ Bis-Tris Protein Gels. Proteins were transferred to PVDF membrane, blocked with $5 \%$ non-fat dry milk (NFDM) in Tris-buffered saline containing $0.1 \%$ Tween 20 (TBST) for $1 \mathrm{~h}$ at room temperature and probed overnight with primary antibodies in $5 \%$ bovine serum albumin in TBST at $4{ }^{\circ} \mathrm{C}$ (under constant shaking on a rocker). After washing three times in TBST for 5 min each, the membrane was incubated with secondary antibodies at 1:5,000 dilution in 5\% NFDM/TBST for $1 \mathrm{~h}$ at room temperature. Membranes were washed three times in TBST, developed with Clarity Western ECL Substrate (Thermo Fisher Scientific). Images have been cropped for presentation (uncropped scans of the blots were shown in Source Data file). 
RNA-seq experiments and analysis. RPE- 1 cells were starved for $12 \mathrm{~h}$ and then transfected with control siRNA or LPAR1 siRNAs, respectively. Following $48 \mathrm{~h}$ serum starvation, cells of siCtrl group were collected (siCtrl LPA $0 \mathrm{~h}$ ), and siCtrl or siLPAR1 cells were both treated with medium contained $2 \mu \mathrm{M}$ LPA for $18 \mathrm{~h}$ (siCtrl LPA $18 \mathrm{~h}$ and siLPAR1 LPA $18 \mathrm{~h}$ ). Total RNAs with polyA tails of these three groups of RPE-1 cells were purified, reverse-transcribed to CDNA, and sequenced. Sequenced reads for each sample were aligned to hg38 with STAR 2.6.0 $\mathrm{a}^{71}$ and differentially expressed genes (DEGs) were identified with DESeq $2^{72}$. We compared samples between siCtrl LPA $18 \mathrm{~h}$ and siCtrl LPA $0 \mathrm{~h}$ groups or siLPAR1 LPA $18 \mathrm{~h}$ and siCtrl LPA $18 \mathrm{~h}$ groups, and DEGs were filtered with $p_{\text {adj }}<0.05$ and $\mid \log 2$ (fold change) $\mid>1$. DAVID ${ }^{73}$ (v6.8, https://david.ncifcrf.gov/) was used to perform gene functional annotation clustering (Gene Ontology: GOTERM_BP_FAT; Pathway: KEGG_PATHWAY).

Thymidine analogs injection. Solutions of $1 \mathrm{mg} / \mathrm{mL} 5$-ethynyl-2'-deoxyuridine (EdU) (Thermo Fisher Scientific) and $10 \mathrm{mg} / \mathrm{mL}$ bromo-deoxyuridine (BrdU) (Thermo Fisher Scientific) were prepared in PBS solution, pH 7.2. BrdU (50 mg/kg) and $\operatorname{EdU}(5 \mathrm{mg} / \mathrm{kg})$ were injected intraperitoneally into the dam mice at $24 \mathrm{~h}$ and $2 \mathrm{~h}$, respectively, before euthanasia.

RNAscope in situ hybridization. We used an RNAscope Multiplex Fluorescent Reagent Kit (320850, ACDbio) and Lpar1 target probes (318501, ACDbio) or Atx target probes (402441-C3, ACDbio) for mouse in situ hybridization of Lparl or Atx mRNA. The experiment was performed according to the manufacturer's instructions.

Isolation and culture of NPCs in cerebral cortex. The cerebral cortexes were dissected out from P0 Lpar1 $1^{+/+}$or Lpar1 $1^{-/-}$embryos. NPCs and other cells in the cortex were isolated in accordance with the manufacturer's instructions (130-092628 , Miltenyi Biotec). Isolated cells were resuspended in Dulbecco's modified Eagle medium (DMEM) containing glutamine, penicillin/streptomycin, sodium pyruvate, $\mathrm{L}$-cysteine and B27, and plated onto coverslips coated with poly-lysine in 24well plates ${ }^{56}$. The cultures were maintained at $37^{\circ} \mathrm{C}$ with $5 \% \mathrm{CO}_{2}$. Cells were then infected with lentivirus expressing GFP or GFP-Lpar1 (titer of $2 \times 10^{6} \mathrm{TU} / \mathrm{mL}$ ) with or without $2 \mu \mathrm{M}$ tubacin treatment. About $72 \mathrm{~h}$ later, cells were fixed and analyzed for cilia length and cell cycle procession.

Statistics and reproducibility. Statistical calculations were performed with SPSS 21.0 software. The Shapiro-Wilk test was used to test the normality and Levene's test was applied to test the homogeneity of variance. For the data of two groups that meet the normal distribution, unpaired two-tailed $t$-test was applied for the comparisons with siCtrl, 0\% FBS control or as indicated in figure legends. For the data of two groups that do not meet the normal distribution, we used

Mann-Whitney test. Multiple comparisons were carried out by using one-way or two-way ANOVA followed by Bonferroni's or Dunnett's multiple comparisons, as noted in the figure legends. For all tests, differences were considered statistically significant if $P$-values were less than 0.05 (as indicated with ${ }^{*},{ }^{*} P<0.05$; ${ }^{*} P<0.01$; $* * * P<0.001)$. No statistical methods were used to predetermine sample size. The experiments were not randomized. No samples were excluded. The investigators were blinded to assess all the staining assays.

Reporting summary. Further information on research design is available in the Nature Research Reporting Summary linked to this article.

\section{Data availability}

All RNA sequencing files were deposited in the short read sequence archive under BioProject ID PRJNA678549. Differentially expressed genes were also mapped to the KEGG database (https://www.kegg.jp/) for pathway analysis. The authors declare that all data supporting the findings of this study are available within the article and its Supplementary Information files, or from the corresponding authors upon reasonable request. Source data are provided with this paper.

Received: 17 December 2019; Accepted: 15 December 2020; Published online: 28 January 2021

\section{References}

1. Gerdes, J. M., Davis, E. E. \& Katsanis, N. The vertebrate primary cilium in development, homeostasis, and disease. Cell 137, 32-45 (2009).

2. Satir, P., Pedersen, L. B. \& Christensen, S. T. The primary cilium at a glance. J. Cell Sci. 123, 499-503 (2010).

3. Huangfu, D. et al. Hedgehog signalling in the mouse requires intraflagellar transport proteins. Nature 426, 83-87 (2003)

4. Rohatgi, R., Milenkovic, L. \& Scott, M. P. Patched1 regulates hedgehog signaling at the primary cilium. Science 317, 372-376 (2007).
5. Corbit, K. C. et al. Kif3a constrains beta-catenin-dependent Wnt signalling through dual ciliary and non-ciliary mechanisms. Nat. Cell Biol. 10, 70-76 (2008).

6. Grisanti, L., Revenkova, E., Gordon, R. E. \& Iomini, C. Primary cilia maintain corneal epithelial homeostasis by regulation of the Notch signaling pathway. Development 143, 2160-2171 (2016)

7. Wong, S. Y. et al. Primary cilia can both mediate and suppress Hedgehog pathway-dependent tumorigenesis. Nat. Med. 15, 1055-1061 (2009).

8. Mykytyn, K. et al. Identification of the gene (BBS1) most commonly involved in Bardet-Biedl syndrome, a complex human obesity syndrome. Nat. Genet. 31, 435-438 (2002)

9. Pazour, G. J., San Agustin, J. T., Follit, J. A., Rosenbaum, J. L. \& Witman, G. B. Polycystin-2 localizes to kidney cilia and the ciliary level is elevated in orpk mice with polycystic kidney disease. Curr. Biol. 12, R378-R380 (2002).

10. Smith, U. M. et al. The transmembrane protein meckelin (MKS3) is mutated in Meckel-Gruber syndrome and the wpk rat. Nat. Genet. 38, 191-196 (2006).

11. Delous, M. et al. The ciliary gene RPGRIP1L is mutated in cerebello-oculorenal syndrome (Joubert syndrome type B) and Meckel syndrome. Nat. Genet. 39, 875-881 (2007).

12. Sanchez, I. \& Dynlacht, B. D. Cilium assembly and disassembly. Nat. Cell Biol. 18, 711-717 (2016)

13. Pugacheva, E. N., Jablonski, S. A., Hartman, T. R., Henske, E. P. \& Golemis, E A. HEF1-dependent Aurora A activation induces disassembly of the primary cilium. Cell 129, 1351-1363 (2007).

14. Kim, S., Lee, K., Choi, J. H., Ringstad, N. \& Dynlacht, B. D. Nek2 activation of Kif24 ensures cilium disassembly during the cell cycle. Nat. Commun. 6, 8087 (2015)

15. Wang, G. et al. PCM1 recruits Plk1 to the pericentriolar matrix to promote primary cilia disassembly before mitotic entry. J. Cell Sci. 126, 1355-1365 (2013).

16. Nielsen, B. S. et al. PDGFRbeta and oncogenic mutant PDGFRalpha D842V promote disassembly of primary cilia through a PLCgamma- and AURKAdependent mechanism. J. Cell Sci. 128, 3543-3549 (2015).

17. Yeh, C. et al. IGF-1 activates a cilium-localized noncanonical Gbetagamma signaling pathway that regulates cell-cycle progression. Dev. Cell 26, 358-368 (2013).

18. Kopinke, D., Roberson, E. C. \& Reiter, J. F. Ciliary hedgehog signaling restricts injury-induced adipogenesis. Cell 170, 340-351 e312 (2017).

19. Willaredt, M. A. et al. A crucial role for primary cilia in cortical morphogenesis. J. Neurosci. 28, 12887-12900 (2008).

20. Marin, O. \& Rubenstein, J. L. A long, remarkable journey: tangential migration in the telencephalon. Nat. Rev. Neurosci. 2, 780-790 (2001).

21. Anthony, T. E., Klein, C., Fishell, G. \& Heintz, N. Radial glia serve as neuronal progenitors in all regions of the central nervous system. Neuron 41, 881-890 (2004).

22. Kowalczyk, T. et al. Intermediate neuronal progenitors (basal progenitors) produce pyramidal-projection neurons for all layers of cerebral cortex. Cereb. Cortex 19, 2439-2450 (2009).

23. Tramontin, A. D., Garcia-Verdugo, J. M., Lim, D. A. \& Alvarez-Buylla, A. Postnatal development of radial glia and the ventricular zone (VZ): a continuum of the neural stem cell compartment. Cereb. Cortex 13, 580-587 (2003).

24. Louvi, A. \& Grove, E. A. Cilia in the CNS: the quiet organelle claims center stage. Neuron 69, 1046-1060 (2011).

25. Uzquiano, A. et al. Mutations in the heterotopia gene Eml1/EML1 severely disrupt the formation of primary cilia. Cell Rep. 28, 1596-1611 e1510 (2019).

26. Seeley, E. S. \& Nachury, M. V. The perennial organelle: assembly and disassembly of the primary cilium. J. Cell Sci. 123, 511-518 (2010).

27. Laguesse, S., Peyre, E. \& Nguyen, L. Progenitor genealogy in the developing cerebral cortex. Cell Tissue Res. 359, 17-32 (2015).

28. Paridaen, J. T., Wilsch-Brauninger, M. \& Huttner, W. B. Asymmetric inheritance of centrosome-associated primary cilium membrane directs ciliogenesis after cell division. Cell 155, 333-344 (2013).

29. Huttner, W. B. \& Kosodo, Y. Symmetric versus asymmetric cell division during neurogenesis in the developing vertebrate central nervous system. Curr. Opin. Cell Biol. 17, 648-657 (2005).

30. Tanaka, M. et al. Autotaxin stabilizes blood vessels and is required for embryonic vasculature by producing lysophosphatidic acid. J. Biol. Chem. 281, 25822-25830 (2006)

31. van Meeteren, L. A. et al. Autotaxin, a secreted lysophospholipase D, is essential for blood vessel formation during development. Mol. Cell. Biol. 26 5015-5022 (2006).

32. Contos, J. J., Fukushima, N., Weiner, J. A., Kaushal, D. \& Chun, J. Requirement for the lpA1 lysophosphatidic acid receptor gene in normal suckling behavior. Proc. Natl Acad. Sci. USA 97, 13384-13389 (2000).

33. Ye, X. et al. LPA3-mediated lysophosphatidic acid signalling in embryo implantation and spacing. Nature 435, 104-108 (2005).

34. Ye, X., Skinner, M. K., Kennedy, G. \& Chun, J. Age-dependent loss of sperm production in mice via impaired lysophosphatidic acid signaling. Biol. Reprod. 79, 328-336 (2008). 
35. Kanda, H. et al. Autotaxin, an ectoenzyme that produces lysophosphatidic acid, promotes the entry of lymphocytes into secondary lymphoid organs. Nat. Immunol. 9, 415-423 (2008).

36. Moolenaar, W. H., van Meeteren, L. A. \& Giepmans, B. N. The ins and outs of lysophosphatidic acid signaling. BioEssays 26, 870-881 (2004).

37. Choi, J. W. et al. LPA receptors: subtypes and biological actions. Annu. Rev. Pharmacol. Toxicol. 50, 157-186 (2010).

38. Liu, S. et al. Expression of autotaxin and lysophosphatidic acid receptors increases mammary tumorigenesis, invasion, and metastases. Cancer Cell 15, 539-550 (2009).

39. Kishimoto, T., Soda, Y., Matsuyama, Y. \& Mizuno, K. An enzymatic assay for lysophosphatidylcholine concentration in human serum and plasma. Clin. Biochem. 35, 411-416 (2002).

40. Tokumura, A. et al. Production of lysophosphatidic acids by lysophospholipase D in human follicular fluids of In vitro fertilization patients. Biol. Reprod. 61, 195-199 (1999).

41. Yung, Y. C., Stoddard, N. C. \& Chun, J. LPA receptor signaling: pharmacology, physiology, and pathophysiology. J. Lipid Res. 55, 1192-1214 (2014).

42. Ohta, H. et al. Ki16425, a subtype-selective antagonist for EDG-family lysophosphatidic acid receptors. Mol. Pharmacol. 64, 994-1005 (2003).

43. Walia, V. et al. Akt Regulates a Rab11-Effector Switch Required for Ciliogenesis. Dev. Cell 50, 229-246 (2019).

44. Plotnikova, O. V. et al. Calmodulin activation of Aurora-A kinase (AURKA) is required during ciliary disassembly and in mitosis. Mol. Biol. Cell 23, 2658-2670 (2012).

45. Inoko, A. et al. Trichoplein and Aurora A block aberrant primary cilia assembly in proliferating cells. J. Cell Biol. 197, 391-405 (2012).

46. Yu, F. X. et al. Regulation of the Hippo-YAP pathway by G-protein-coupled receptor signaling. Cell 150, 780-791 (2012).

47. Kim, J. et al. Actin remodelling factors control ciliogenesis by regulating YAP/ TAZ activity and vesicle trafficking. Nat. Commun. 6, 6781 (2015).

48. Jans, R. et al. Lysophosphatidic acid promotes cell migration through STIM1and Orail-mediated Ca2+(i) mobilization and NFAT2 activation. J. Investig. Dermatol. 133, 793-802 (2013).

49. Ohata, H., Yamada, H. \& Momose, K. Lysophosphatidic acid induces shear stress-dependent $\mathrm{Ca}^{2+}$ influx in mouse aortic endothelial cells in situ. Exp. Physiol. 96, 468-475 (2011).

50. Todorova, M. G., Fuentes, E., Soria, B., Nadal, A. \& Quesada, I. Lysophosphatidic acid induces $\mathrm{Ca}^{2+}$ mobilization and c-Myc expression in mouse embryonic stem cells via the phospholipase C pathway. Cell Signal 21, 523-528 (2009).

51. Hecht, J. H., Weiner, J. A., Post, S. R. \& Chun, J. Ventricular zone gene-1 (vzg1) encodes a lysophosphatidic acid receptor expressed in neurogenic regions of the developing cerebral cortex. J. Cell Biol. 135, 1071-1083 (1996).

52. Sato, K. et al. Identification of autotaxin as a neurite retraction-inducing factor of PC12 cells in cerebrospinal fluid and its possible sources. J. Neurochem. 92, 904-914 (2005).

53. Estivill-Torrus, G. et al. Absence of LPA1 signaling results in defective cortical development. Cereb. Cortex 18, 938-950 (2008).

54. Kostic, M. et al. YAP activity is necessary and sufficient for basal progenitor abundance and proliferation in the developing neocortex. Cell Rep. 27, 1103 (2019).

55. Park, R. et al. Yap is required for ependymal integrity and is suppressed in LPA-induced hydrocephalus. Nat. Commun. 7, 10329-10329 (2016).

56. Shao, W. et al. Centrosome anchoring regulates progenitor properties and cortical formation. Nature 580, 106-112 (2020).

57. Tsang, W. Y. \& Dynlacht, B. D. CP110 and its network of partners coordinately regulate cilia assembly. Cilia 2, 9 (2013).

58. Lai, S. L. et al. Autotaxin/Lpar3 signaling regulates Kupffer's vesicle formation and left-right asymmetry in zebrafish. Development 139, 4439-4448 (2012).

59. Frisca, F. et al. Role of ectonucleotide pyrophosphatase/phosphodiesterase 2 in the midline axis formation of zebrafish. Sci. Rep. 6, 37678 (2016).

60. Wang, L. \& Dynlacht, B. D. The regulation of cilium assembly and disassembly in development and disease. Development 145, 18 (2018).

61. Kinzel, D. et al. Pitchfork regulates primary cilia disassembly and left-right asymmetry. Dev. Cell 19, 66-77 (2010).

62. Lee, K. H. et al. Identification of a novel Wnt5a-CK1varepsilon-Dvl2-Plk1mediated primary cilia disassembly pathway. EMBO J. 31, 3104-3117 (2012).

63. Huang, X. et al. Transventricular delivery of Sonic hedgehog is essential to cerebellar ventricular zone development. Proc. Natl Acad. Sci. USA 107, 8422-8427 (2010).

64. Lehtinen, M. K. et al. The cerebrospinal fluid provides a proliferative niche for neural progenitor cells. Neuron 69, 893-905 (2011).
65. Ding, W., Wu, Q., Sun, L., Pan, N. C. \& Wang, X. Cenpj regulates cilia disassembly and neurogenesis in the developing mouse cortex. J. Neurosci. 39, 1994-2010 (2019)

66. Mills, G. B. \& Moolenaar, W. H. The emerging role of lysophosphatidic acid in cancer. Nat. Rev. Cancer 3, 582-591 (2003).

67. Houben, A. J. \& Moolenaar, W. H. Autotaxin and LPA receptor signaling in cancer. Cancer Metastasis Rev. 30, 557-565 (2011).

68. Sutphen, R. et al. Lysophospholipids are potential biomarkers of ovarian cancer. Cancer Epidemiol. Biomark. Prev.13, 1185-1191 (2004).

69. Wang, P., Wu, X., Chen, W., Liu, J. \& Wang, X. The lysophosphatidic acid (LPA) receptors their expression and significance in epithelial ovarian neoplasms. Gynecologic Oncol. 104, 714-720 (2007).

70. Sun, K. et al. Aberrant expression and potential therapeutic target of lysophosphatidic acid receptor 3 in triple-negative breast cancers. Clin. Exp. Med. 15, 371-380 (2015).

71. Dobin, A. et al. STAR: ultrafast universal RNA-seq aligner. Bioinformatics 29, 15-21 (2013)

72. Love, M. I., Huber, W. \& Anders, S. Moderated estimation of fold change and dispersion for RNA-seq data with DESeq2. Genome Biol. 15, 550 (2014).

73. Jiao, X. et al. DAVID-WS: a stateful web service to facilitate gene/protein list analysis. Bioinformatics 28, 1805-1806 (2012).

\section{Acknowledgements}

We would like to thank Professor Xue-liang Zhu, Faxing Yu, and Jiahuai Han for providing cells, plasmids, antibodies and technique support. This work was also funded by the National Basic Research Program of China, (2014CB910603), National Natural Science Foundation of China, (No. 81521064; No. 81790252). National key research and development program, (2017YFC1601100; 2017YFC1601101; 2017YFC1601102; 2017YFC1601104). National Major Scientific and Technological Special Project for "Significant New Drugs Development".

\section{Author contributions}

H.-Y.L. and X.-M.Z. supervised the project; H.-B.H. and Z.-Q.S. designed and carried out most of the experiments; J.C. provided mice and suggestions; G.-P.S, S.L., X.-L.S., J.-F.Y., T.-T.L., H.-Q.T. and Y.-C.Z. analyzed the data; Y.-L.X., M.W., P.-Y.L., Q.-Y.H., A.-L.L. and T.Z. carried out the statistics; H.-B.H. and Z.-Q.S., H.-Y.L. and X.-M.Z. wrote the paper. All authors discussed the results and commented on the manuscript.

\section{Competing interests}

The authors declare no competing interests.

\section{Additional information}

Supplementary information The online version contains supplementary material available at https://doi.org/10.1038/s41467-021-20986-y.

Correspondence and requests for materials should be addressed to X.-M.Z. or H.-Y.L.

Peer review information: Nature Communications thanks the anonymous reviewers for their contribution to the peer review of this work.

Reprints and permission information is available at http://www.nature.com/reprints

Publisher's note Springer Nature remains neutral with regard to jurisdictional claims in published maps and institutional affiliations.

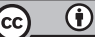

Open Access This article is licensed under a Creative Commons Attribution 4.0 International License, which permits use, sharing, adaptation, distribution and reproduction in any medium or format, as long as you give appropriate credit to the original author(s) and the source, provide a link to the Creative Commons license, and indicate if changes were made. The images or other third party material in this article are included in the article's Creative Commons license, unless indicated otherwise in a credit line to the material. If material is not included in the article's Creative Commons license and your intended use is not permitted by statutory regulation or exceeds the permitted use, you will need to obtain permission directly from the copyright holder. To view a copy of this license, visit http://creativecommons.org/ licenses/by/4.0/.

(C) The Author(s) 2021 